\title{
A sequential implicit algorithm of chemo-thermo-poro-mechanics for fractured geothermal reservoirs
}

\author{
Jihoon Kim ${ }^{\mathrm{a}, 1}$, Eric Sonnenthal ${ }^{\mathrm{a}}$, Jonny Rutqvist ${ }^{\mathrm{a}}$ \\ ${ }^{a}$ Earth Sciences Division, Lawrence Berkeley National Laboratory. 1 Cyclotron Road 74R316C, Berkeley, CA 94720, USA \\ ${ }^{b}$ Harold Vance Department of Petroleum Engineering, Texas A\&M University. 3116 TAMU 407K Richardson Building College Station, TX 77843, \\ USA
}

\begin{abstract}
We describe the development of a sequential implicit formulation and algorithm for coupling fluid-heat flow, reactive transport, and geomechanics. We consider changes in pore volume from dissolution caused by chemical reactions, in addition to coupled flow and geomechanics. Moreover, we use the constitutive equations for the multiple porosity model for fractured geothermal reservoirs, employing failure-dependent permeability dynamically and updating it every time step. The proposed sequential algorithm is an extension of the fixed-stress split method to chemo-thermoporo-mechanics, facilitating the use of existing flow-reactive transport and geomechanics simulators.

We first validate a simulator that employs the proposed sequential algorithm, matching the numerical solutions with the analytical solutions such as Terzaghi's and Mandel's problems for poro-mechanics and the reference solutions of chemo-poro-mechanics and chemo-thermo-poro-mechanics in the 1D elastic problems. We also perform convergence test, and the proposed algorithm shows fast convergence, when full iteration is taken, and first order accuracy in time for the staggered approach.

We then investigate two test cases: 2D multiple porosity elastic and 3D single porosity elastoplastic problems, and explore the differences in coupled flow and geomechanics with and without reactive transport. We find that the change in pore-volume induced by mineral dissolution can impact on fluid pressure and failure status, followed by significant changes in permeability and flow variables, showing strong interrelations between flow-reactive transport and geomechanics.
\end{abstract}

Keywords: Poromechanics, Fractured geothermal reservoirs, Reactive transport, Multiple interacting continua (MINC), Shear stimulation

Email address: JihoonKim@lbl.gov; Tel.:+1 510-486-5793 (Jihoon Kim) 


\section{Introduction}

Enhanced geothermal reservoirs that exhibit increased permeability resulting from shear stimulation of a fracture volume can provide large heat extraction accompanied by economic flow rates and energy production. Fractures are highly compressible compared with intact rock, so they may affect overall geomechanical responses significantly, even though they occupy a smaller bulk volume (Bai, 1999). Permeability can significantly be affected by deformation of fractures through the relationship between the fracture aperture and permeability (Snow, 1965; Rutqvist and Stephansson, 2003). Furthermore, chemical or thermal nonequilibrium between injection fluid and the reservoir host rock can result in dissolution and precipitation, changing the porosity (Xu et al., 2011). Such changes in pore volume can change fluid pressure, and hence effective stress and strain. Permeability changes through mineral-water reactions and geomechanics affect solute transport which can lead to strong thermal, hydrological, mechanical, and chemical coupling. These physical processes occur simultaneously, but they have different time scales. The geomechanical problem is written as the elliptic partial differential equation (PDE) for modeling quasi-static mechanics, showing the instantaneous mechanical responses (Coussy, 1995; Lewis and Schrefler, 1998), while pressure and saturation equations of the multiphase flow problems are expressed as the parabolic and hyperbolic PDEs, which model time-dependant physical behavior (Aziz and Settari, 1979; Pruess et al., 1999). Reaction between fluid and the porous media can be modeled based on either a rate-independent equation from local equilibrium assumption or a rate-dependent form of kinetic reaction (Moridis et al., 2008; Xu et al., 2008). The different time scales and strong couplings in those physics result in high numeral stiffness and strong nonlinearity, when we solve the coupled mathematical equations numerically, thus robust and rigorous numerical modeling is necessary for accuracy.

The geothermal reservoirs are naturally or artificially fractured for economic feasibility, consisting of the fracture(s) and rock matrix. The multiple continuum approach is a widely used approach that can represent the fracture-rock matrix systems consisting of a highly permeable fracture contimuum transporting fluid over the domain, while the matrix stores fluid and conveys it to the highly permeable fracture continuum (Barenblatt et al., 1960; Berryman, 2002; Sonnenthal et al., 2005; Taron et al., 2009). In geothermal reservoirs, we may introduce more than two continua (e.g., representing fracture and several rock matrix continua) for more accurate modeling of heat flow. Recently, Kim et al. (2012) proposed formulation and sequential numerical algorithms for coupled fluid-heat flow and geomechanics within such a multiple continuum approach. We have implemented these functionalities within ROCMECH, an in- 
house geomechanics simulator, which is coupled to TOUGH family codes, flow simulators.

The study presented in this paper is based on the latter study, employing nonlinear dynamic strain and failuredependent permeability and dynamic geomechanical moduli in order to capture complex interactions between flow and geomechanics. In this study, we consider changes in pore volume, induced by chemical reaction, when solving for fluid and heat flow. When combining the two simulators, we solve fluid and heat flow, geomechanics, and reactive transport problems sequentially, adopting implicit solution schemes for the subproblems (i.e., sequential implicit method). Specifically, when solving flow and geomechanics, we employ the fixed-stress sequential method, which solves flow first, fixing the total stress field locally, and solves geomechanics at the next step from the calculated flow solutions. The fixed-stress method can provide numerical unconditional stability and high accuracy, comparable to the fully implicit method, regardless of the coupling strength in pore volume between flow and geomechanics (Kim et al., 2011b, 2013, 2012). Taron et al. (2009) employed the undrained sequential method, which solves geomechanics first, fixing fluid mass locally, and then solves flow at the next step. The undrained sequential method can also provide numerical stability in solving flow and geomechanics (Armero, 1999), but causes inaccuracy or non-convergence for the high coupling strength in the case of highly deformable porous media (e.g., fracture) and incompressible fluid

(e.g. liquid water) (Kim et al., 2011a). The fixed-stress method can easily be implemented by using the porosity function and its correction (Settari and Mourits, 1998; Kim et al., 2012). In this study, we incorporate the porosity change from chemical reaction into the sequentially coupled simulator, TOUGHREACT+ROCMECH (shortly, T+M). In $\mathrm{T}+\mathrm{M}$, flow-geomechanics properties and variables (e.g., porosity, permeability, fluid-phase pressure and saturation, displacement, effective stress, geomechanical moduli, failure status) are updated dynamically along with the transient coupled simulation. We perform several verification test cases for $\mathrm{T}+\mathrm{M}$ (e.g., the Terzaghi and Mandel problems, 1D elastic chemo-poro-mechanics and chemo-thermo-poro-mechanics problems), as well as their convergence analysis. Then, we investigate 2D and 3D geothermal problems, using coupled thermo-poro-mechanics simulations with and without reactive transport, and analyze differences between them.

\section{Mathematical description}

We briefly describe the governing equations of geomechanics, fluid and heat flow with reactive solute transport. Next, we introduce constitutive equations for the multiple porosity model and the changes in pore volume from geomechanics and chemical reactions. 


\subsection{Governing Equations}

The governing equation for geomechanics is based on the quasi-static assumption, written as (Coussy, 1995)

$$
\operatorname{Div} \boldsymbol{\sigma}+\rho_{b} \boldsymbol{g}=\mathbf{0},
$$

where Div is the divergence operator, $\boldsymbol{\sigma}$ is the total stress tensor, $\rho_{b}$ is the bulk density, and $\boldsymbol{g}$ is the gravity vector. The infinitesimal transformation is used to allow the strain tensor, $\varepsilon$, to be the symmetric gradient of the displacement vector, $\boldsymbol{u}$

$$
\varepsilon=\frac{1}{2}\left(\operatorname{Grad} \boldsymbol{u}+\operatorname{Grad}^{t} \boldsymbol{u}\right),
$$

57 67 liquid, $\mathbf{w}_{J}^{k}$ is described by Darcy's law as

$$
\mathbf{w}_{J}^{k}=\psi_{J}^{k} \mathbf{w}_{J} \quad \mathbf{w}_{J}=-\frac{\rho_{J} k_{r J}}{\mu_{J}} \mathbf{k}_{p}\left(\operatorname{Grad} P_{J}-\rho_{J} \boldsymbol{g}\right),
$$

$$
\frac{d}{d t} \int_{\Omega} m^{k} d \Omega+\int_{\Gamma} \mathbf{f}^{k} \cdot \mathbf{n} d \Gamma=\int_{\Omega} q^{k} d \Omega
$$
normal vector to the boundary.

The mass flow term $\mathbf{f}^{k}$ in Equation 3 is given by

$$
\mathbf{f}^{k}=\sum_{J}\left(\mathbf{w}_{J}^{k}+\mathbf{J}_{J}^{k}\right)
$$
the case that the fluid is gaseous, $\mathbf{w}_{J}^{k}$ can be written as

where the superscript $k$ indicates the fluid component or heat. $d(\cdot) / d t$ means the time derivative of a physical quantity $(\cdot)$ relative to the motion of the solid skeleton. $m^{k}$ is mass of component $k$ or heat, where the superscript $(\cdot)^{\theta}$ indicates heat. $\mathbf{f}^{k}$ and $q^{k}$ are its flux and source terms on the domain $\Omega$ with a boundary surface $\Gamma$, respectively, where $\mathbf{n}$ is the

where $\mathbf{w}_{J}^{k}$ and $\mathbf{J}_{J}^{k}$ are the convective and diffusive mass flows of component $k$ in the phase $J$. When the fluid phase is

where $\mathbf{k}_{p}$ is the second order absolute permeability tensor; $\rho_{J}, \mu_{J}, k_{r J}$, and $P_{J}$ are the density, viscosity, relative permeability, and pressure of the fluid phase $J$, respectively. $\psi_{J}^{k}$ is the mass fraction of component $k$ in the phase $J$. In

$$
\mathbf{w}_{J}=-\left(1+\frac{k_{K}}{P_{J}}\right) \mathbf{k}_{p} \frac{\rho_{J} k_{r J}}{\mu_{J}}\left(\operatorname{Grad} P_{J}-\rho_{J} \boldsymbol{g}\right),
$$


71 where $k_{K}$ is the Klinkenberg factor (Klinkenberg, 1941). The diffusive flow $\mathbf{J}_{J}^{k}$ is described as

$$
\mathbf{J}_{k}^{J}=-\Phi S_{J} \tau_{G} \mathbf{D}_{J}^{k} \rho_{J} \mathbf{G r a d} \psi_{J}^{k}
$$

72

73

where $\mathbf{D}_{J}^{k}$ is the second order hydrodynamic dispersion tensor of component $k$ in the phase $J$, and $\tau_{G}$ is the gas tortuosity. $\Phi$ (Lagrange's porosity, also called reservoir porosity) is defined as the ratio of the pore volume in the deformed configuration to the bulk volume in the reference (typically initial) configuration. $S_{J}$ is the saturation of the fluid phase $J$.

The heat flux, $f^{\theta}$, is obtained by heat conduction and convection laws, written as

$$
\boldsymbol{f}^{\theta}=-\left((1-\Phi) \mathbf{K}_{R}+\Phi S_{J} \mathbf{K}_{J}\right) \mathbf{G r a d} T+h_{J} \boldsymbol{w}_{J}
$$

where $\mathbf{K}_{R}$ and $\mathbf{K}_{J}$ are the second order thermal conductivity tensors of the porous media and fluid phase $J$, respectively. $T$ is temperature. $h_{J}$ is the specific enthalpy of phase $J$.

The governing equation for solute transport with chemical reaction (i.e., reactive transport) is obtained from mass conservation (Xu et al., 2011), described as

$$
\frac{d}{d t}\left(\Phi C_{i}\right)+\operatorname{Div}\left(\Phi \boldsymbol{v}_{i} C_{i}-\Phi \mathbf{D}_{i} \mathbf{G r a d} C_{i}\right)=-\sum_{m=1}^{n_{c}} \gamma_{i m} r_{m}+q_{i}
$$

where $C_{i}$ is the concentration of the $\mathrm{i}^{\text {th }}$ chemical. $\boldsymbol{v}_{i}$ and $\mathbf{D}_{i}$ are the Darcy velocity vector and diffusion coefficient matrix of the $\mathrm{i}^{\text {th }}$ chemical, respectively. $\gamma_{i m}$ and $r_{m}$ are the stoichiometric coefficient of the $\mathrm{i}^{\text {th }}$ chemical in the $\mathrm{m}^{\text {th }}$ chemical and the reaction rate of the $\mathrm{m}^{\text {th }}$ chemical, respectively. $n_{c}$ is the total number of the chemicals, and $q_{i}$ is the possible external source of the $\mathrm{i}^{\text {th }}$ chemical.

\subsection{Constitutive relations in coupled flow and geomechanics}

The constitutive relations for thermoporomechanics in a single porosity model are originally based on the Biot theory (e.g., Biot (1941); Biot and Willis (1957); Geertsma (1957); Coussy (1995)). In this study, we employ the constitutive equations proposed by Kim et al. (2012) for the multiple porosity model (Figure 1), developed from the isothermal double porosity elastic model (Berryman, 2002) into the nonisothermal multiple porosity elastoplastic 


$$
\begin{aligned}
\delta \boldsymbol{\sigma}= & \overbrace{\mathbf{C}_{u p}: \delta \underbrace{\delta \sigma^{\prime}}_{\varepsilon_{e}}}^{\left(\varepsilon-\varepsilon_{p}\right)}-b_{l, J}^{*} \delta p_{l, J} \mathbf{1}-K_{d r} \tilde{b}_{l} \delta T \mathbf{1}, b_{l, J}^{*}=-K_{d r}\left(b S_{J}\right)_{l}, \\
& \frac{1}{K_{d r}}=\frac{\eta_{k}}{K_{k}}, \mathbf{C}_{u p}=K_{d r}\left(\frac{\eta}{K}\right)_{k} \mathbf{C}_{k}, b_{l}=-\left(\frac{\alpha \eta}{K}\right)_{l}, \tilde{b}_{l}=3\left(\alpha_{T} \eta\right)_{l}, \\
\delta \zeta_{(l, J)}= & b_{l, J}^{*} \delta \varepsilon_{v}+L_{l, J, m, I}^{-1} \delta p_{m, I}-\bar{D}_{l, J, m} \delta T_{m}, \\
\left(\bar{S}-\bar{s}_{J} \delta m_{J}\right)_{l}= & -\tilde{b}_{l} K_{d r} \delta \varepsilon_{v}-\bar{D}_{l, m, I} \delta p_{m, I}+\tilde{D}_{l, m} \delta T_{m},
\end{aligned}
$$

where double indices indicate summation. The subscript $l$ indicates a material (sub-element) within a gridblock. $\delta$ implies variation relative to the motion of the solid skeleton. $\boldsymbol{\sigma}^{\prime}, K_{d r}$, and $\mathbf{C}_{u p}$ are the effective stress tensor, drained bulk modulus, and elasticity tensor at the level of the gridblock, respectively. $\mathbf{1}$ is the rank-2 identity tensor. $\delta \zeta_{(l, J)}=\delta m_{(l, J)} / \rho_{(l, J)}$, where $\delta m_{(l, J)}$ and $\rho_{(l, J)}$ are mass and density of fluid phase $J$ in the material $l$, respectively. $\varepsilon_{v}$ is the total volumetric strain. $b_{l, J}^{*}$ and $\tilde{b}_{l}$ are the coupling coefficients. $\alpha_{l}, \alpha_{(T, l)}, \eta_{l}$, and $K_{l}$ are the Biot coefficient, thermal dilation coefficient, volume fraction, and drained bulk modulus for material $l$, respectively. $\alpha_{l}=1-K_{l} / K_{l}^{s}$, where $K_{l}^{s}$ is the intrinsic solid grain bulk modulus of material $l . \mathbf{C}_{l}$ is the isothermal drained elasticity tensor for material $l . L_{l, J, m, I}$ represents a modulus for the multiple porous model, similar to the Biot modulus matrix, and readers can refer to Kim et al. (2012) for the specific forms of $L_{l, J, m, I} . \bar{S}_{J}$ is the total entropy, and $\bar{s}_{J}$ is the internal entropy per unit mass of phase $J$ (i.e., specific entropy of phase $J$ ). $\bar{D}_{l, m, I}$ is the coupling coefficient between fluid flow and heat flow based on thermodynamics. $\tilde{D}_{l, m}$ represents relations between temperatures of all materials in the heat flow simulation (i.e., total heat capacity matrix).

The local effective stress and local total strain at each subelement $l\left(\sigma_{l}^{\prime}\right.$ and $\varepsilon_{l}$, respectively) are obtained as

$$
\delta \boldsymbol{\sigma}^{\prime}{ }_{l}=(\mathbf{C}: \delta \boldsymbol{\varepsilon})_{l}, \delta \boldsymbol{\varepsilon}_{l}=\frac{K_{d r}}{K_{k}} \delta \boldsymbol{\varepsilon}, \delta \boldsymbol{\sigma}^{\prime}=\sum_{l=1}^{n_{m}} \eta_{l} \delta \boldsymbol{\sigma}_{l}^{\prime},
$$

where $n_{m}$ is the total number of the materials.

In this study, we neglect the heat contribution (i.e., entropy production) directly from geomechanics to heat flow, ignoring the term related to $-\tilde{b}_{l} K_{d r} \delta \varepsilon_{v}$ of Equation 13. This assumption is justified when heat capacity of material or fluid is high, or when direct heat generation from deformations is negligible (Lewis and Schrefler, 1998). Then, according to Kim et al. (2012), Lagrange's porosity of material $l$ without chemical reaction, $\Phi_{l}^{M}$, can be written as

$$
\delta \Phi_{l}^{M}=\left(\frac{\alpha_{l}^{2}}{K_{l}}+\frac{\alpha_{l}-\Phi_{l}}{K_{s}}\right) \delta p_{l}+3 \alpha_{T, l} \alpha_{l} \delta T_{l}-\frac{b_{l}}{\eta_{l}} \delta \sigma_{v}
$$


where $\sigma_{v}$ is the total (volumetric) mean stress. Equation 15 is typically known as the porosity change, when reservoir simulation is coupled to geomechanics. The additional change in the porosity of material $l$ due to reactive solute transport, $\delta \Phi_{l}^{C}$, can be formulated as (Xu et al., 2006)

$$
\delta \Phi_{l}^{C}=-\sum_{m=1}^{n_{c}} \delta \chi_{m, l}
$$

where $\chi_{m, l}$ is the $\mathrm{m}^{t h}$ solid volume fraction of material $l$. Then, from Equations 15 and 16 , we obtain the total porosity change due to geomechanics and geochemistry, as follows.

$$
\delta \Phi_{l}\left(=\delta \Phi_{l}^{M}+\delta \Phi_{l}^{C}\right)=\left(\frac{\alpha_{l}^{2}}{K_{l}}+\frac{\alpha_{l}-\Phi_{l}}{K_{s}}\right) \delta p_{l}+3 \alpha_{T, l} \alpha_{l} \delta T_{l}-\frac{b_{l}}{\eta_{l}} \delta \sigma_{v}-\sum_{m=1}^{n_{c}} \delta \chi_{m, l} .
$$

\section{Numerical integration}

We use finite volume and finite element methods for flow and geomechanics for space discretization, respectively. As indicated in Kim et al. (2012), this mixed finite volume and finite element formulation has local conservations of mass and heat at the element level for flow, continuous displacement field in geomechanics, and convergent approximations in space with the lowest order discretization. Furthermore, the mixed formulation shows relatively stable approximations because of a piecewise constant interpolation for fluid pressure, compared with the finite element method for flow that is formulated based on a piecewise continuous interpolation for pressure (Vermeer and Verruijt, 1981; Phillips and Wheeler, 2007; Kim et al., 2011a,b). In time discretization, the backward Euler method is used as typically employed in reservoir simulation (Aziz and Settari, 1979). The initial total stress satisfies the geomechanical equilibrium, consistent with the boundary conditions.

In the numerical integration, we use a sequential implicit approach, by which fluid and heat flow, geomechanics, and reactive solute transport are solved sequentially, with each subproblem being solved implicitly, as shown in Figure 2. TOUGHREACT, a coupled flow and reactive solute transport simulator, is also based on the sequential implicit approach (Xu et al., 2008). We develop the modified fixed-stress-split method in coupling between fluid-heat flow and geomechanics for the multiple porosity model, incorporating a chemo-thermo-poro-mechanics approach. Specifically, from Equation 17, we can easily implement the modified fixed stress split by using the porosity function and its correction, as follows.

$$
\Delta \Phi_{l}^{n}=\left(\frac{\alpha_{l}^{2}}{K_{l}}+\frac{\alpha_{l}-\Phi_{l}^{n}}{K_{s}}\right) \Delta p_{l}^{n}+\left(3 \alpha_{T, l} \alpha_{l}\right) \Delta T_{l}^{n}-\frac{b_{l}}{\eta_{l}} \Delta \sigma_{v}^{n-1}+\Delta \Phi_{l}^{C, n-1},
$$


where $\Delta(\cdot)^{n}=(\cdot)^{n+1}-(\cdot)^{n}$. The fixed stress split is unconditionally stable (Von Neumann stability and B-stability

for linear and nonlinear problems, respectively) and accurate in coupled flow and geomechanics without the reactive transport (Kim et al., 2011b, 2013, 2012; Kim and Moridis, 2014). However, unconditional stability of chemo-thermoporo-mechanics has not been proved mathematically, because thermo-poro-mechanics is only a subproblem of chemothermo-poro-mechanics.

Once individual parallel codes for respective flow and geomechanics are available, the proposed algorithm can easily be applied to construct a parallelized coupled simulator, because it only requires simple and local code modification with data communication gridblock by gridblock. Thus, we can keep high scalability if the individual parallel codes are highly scalable.

\section{Numerical examples}

We first conduct tests of verification and numerical convergence for T+M's chemo-thermo-poro-mechanics approach. To this end, we first introduce the Terzaghi and Mandel problems to verify the simulator in poromechanics. Then we compare TOUGHREACT only (TR-only) with T+M for 1D elastic single-phase flow problems, because the 1D elastic problems with no extra external mechanical loading can allow the decoupling of flow and geomechanics, when we use an exact pore compressibility (i.e., $\frac{1}{\Phi} \frac{d \Phi}{d p}$ ) and thermal expansivity (i.e., $\frac{1}{\Phi} \frac{d \Phi}{d T}$ ) in flow. We also perform convergence analysis for these test cases, such as number of iterations for full iteration and accuracy in time. After verification and convergence analysis, we compare the numerical results among chemo-thermo-poro-mechanics, thermo-poro-mechanics without reactive transport, and flow-only with and without reactive transport for naturally and artificially fractured geothermal reservoirs.

\subsection{Verification and numerical convergence 4.1.1. Terzaghi's and Mandel's problems}

Figure 3 shows the domains for the (isothermal) Terzaghi and Mandel problems, in which 2D plane strain geomechanics is employed. For Terzaghi's problem, we have 31 gridblocks, the sizes of which are uniform, $1.0 \mathrm{~m}$. We impose a drainage boundary on the left side and no-flow conditions on the right side. Liquid water is fully saturated, and the initial and boundary pressures are both 8.3 MPa. The initial total stress is also 8.3 MPa over the domain, and we apply a 16.6 MPa normal to the left boundary, two times greater than the initial total stress. The Young's modulus 
and Poisson ratio's are $450 \mathrm{MPa}$ and 0.0, respectively. Only horizontal displacement is allowed. The monitoring well is located at the right end.

For Mandel's problem, we have $40 \times 5$ gridblocks, taking the upper domain by symmetry. The sizes of the gridblocks in the $\mathrm{x}$ direction are uniform, $0.5 \mathrm{~m}$, while the sizes in the $\mathrm{z}$ direction are non-uniform; $0.005 \mathrm{~m}, 0.01 \mathrm{~m}$, $0.05 \mathrm{~m}, 0.1 \mathrm{~m}, 0.1 \mathrm{~m}$. The medium is fully saturated with liquid water, and the initial pressure is $10.0 \mathrm{MPa}$. We impose drainage boundaries at the left and right sides, and no-flow conditions on the other sides. Initial total stress is also 10.0 MPa over the domain, and we set 20.0 MPa for the overburden, two times greater than the initial total stress. The Young's modulus and Poisson's ratio are $450 \mathrm{MPa}$ and 0.0 , respectively. The monitoring well is located in the middle of the domain, as shown in Figure 3. No gravity is considered for the Terzaghi and Mandel problems. The permeability and initial porosity are $6.51 \times 10^{-15} \mathrm{~m}^{2}, 6.6 \mathrm{mD}\left(1 \mathrm{Darcy}=9.87 \times 10^{-13} \mathrm{~m}^{2}\right)$, and 0.425 , respectively for both problems, and the permeability is constant during simulation. Biot's coefficients for both problems are 1.0.

Figure 4 shows that the staggered (i.e., one iteration) and fully iterated solutions from $\mathrm{T}+\mathrm{M}$ match the analytical solutions. The numerical results correctly capture the instantaneous pressure buildup for Terzaghi's problem and the Mandel-Cryer effect for Mandel's problem; these could not be captured by a flow-only simulation. From Figure 5, we can identify fast convergence of the fixed-stress method. When full iteration is taken, the fixed-stress split almost takes 3 iterations for both Terzaghi's and Mandel's problems.

Figures 4 and 5 support the mathematical and numerical analyses in Kim et al. (2011b, 2013). In the analyses, the staggered and full iterative fixed-stress methods yield numerical convergence in time. In addition, the staggered fixed-stress method yields high accuracy and efficiency. Here, convergence in time means that the numerical solution approaches the true solution when time step size is refined, and accuracy is measured by the error between the numerical solution and the true solution.

\subsubsection{D elastic chemo-poro-mechanics}

We reuse the domain, boundary conditions, and initial porosity and permeability used in the Terzaghi problem, while having the same initial stress and left boundary stress as the initial fluid pressure. Initially, liquid water is fully saturated, having 8.3 MPa. The Young's modulus and Poisson's ratio are 2.4 GPa and 0.2, respectively, and Biot's coefficient is 1.0 .

We account for reactive transport with chemical reaction, while isothermal single-phase flow is considered. The 
injection is applied to the right end gridblock at the rate of $1.0 \times 10^{-4} \mathrm{~kg} \cdot \mathrm{s}^{-1}$. Quartz dissolution is assumed to be the only reaction as observed in the experiment described by Johnson et al. (1998), and modeled using a linear kinetic rate law, written as

$$
r_{q z}=-\chi_{q z} A_{q z} \kappa_{q z}\left(1-\frac{C_{S i}}{C_{S i, q z}}\right)
$$

where the subscript $(\cdot)_{q z}$ indicates quartz. $A_{q z}$ and $\kappa_{q z}$ are the specific surface area and the reaction rate constant, respectively. $C_{S i}$ is the concentration of dissolved (aqueous) quartz, and $C_{S i, q z}$ is $C_{S i}$ in equilibrium. $A_{q z}$ is $54.2 \mathrm{~m}^{2} \cdot \mathrm{kg}^{-1}$ (Knauss and Copenhaver, 1995), and $\kappa_{q z}$ is $2.836 \times 10^{-8} \mathrm{~mol} \cdot \mathrm{m}^{-2} \cdot \mathrm{s}^{-1}$ at $240^{\circ} \mathrm{C}$ (Tester et al., 1994). The initial condition of $C_{S i}$ is zero, which is not in chemical equilibrium at initial time, in order to test the pressure change from the dissolution of quartz. $C_{S i}$ of the injection water is also zero. $C_{S i, q z, 0}\left(C_{S i, q z}\right.$ at initial temperature, $240^{\circ} \mathrm{C}$ ) is $6.38 \times 10^{-3} \mathrm{~mol} / \mathrm{kg}$. The initial $\mathrm{pH}$ of water is 5.589 .

Figure 6 (left) shows comparison of the results between TR-only and T+M for the 1D chemo-poro-mechanics problem. TR-only indicates the TOUGHREACT only simulation. As indicated previously, the coupled flow and geomechanics problem of this test can also be modeled by the flow-only simulation, having $0.9804 \times 10^{-9} \mathrm{~Pa}^{-1}$ of the exact pore-compressibility, which is calculated from the constrained geomechanics condition of this test case. In this figure, we can see that the results of $\mathrm{T}+\mathrm{M}$ match the results for TR-only, validating $\mathrm{T}+\mathrm{M}$.

Figure 7 (left) shows that significant quartz dissolution occurs at early time and at the same rate over the entire domain. This dissolution increases pore-volume and $C_{q z}$ rapidly (Figure 7 (right)). The increased pore-volume induces expansion of the pore fluid, liquid water, depending on fluid compressibility. As a result, the fluid pressure decreases over the domain at early time (Figure 6 (left)).

In the left of Figure 6, the pressure drops are at different points over the domain, because there are influxes to the domain, even though the rates of the dissolution are the same over the entire domain. At the left boundary, liquid water flows into the domain due to the constant pressure boundary, whereas at the right boundary, a constant water-injection rate is applied. Thus, the decrease of pressure at early time is low near the both ends (e.g., $x_{d}=0.113(\mathrm{x}=3.5 \mathrm{~m})$ and $\left.x_{d}=0.984(\mathrm{x}=30.5 \mathrm{~m})\right)$, while it is high around the middle of the domain (e.g., $x_{d}=0.597(\mathrm{x}=18.5 \mathrm{~m})$ and $\left.x_{d}=0.758(\mathrm{x}=23.5 \mathrm{~m})\right)$

As $C_{S i}$ increases, as shown in the right of Figure 7, the rate of dissolution decreases, based on the linear kinetic rate law in Equation 18. Then pressure increases again because the effect from the influxes becomes more dominant than 
the dissolution effect. At late time, when the dissolution is tiny, the pressure distribution becomes monotonic without a hump; the highest pressure at the right end (i.e, the injection point), and the lowest pressure at the left end (i.e., the constant pressure boundary).

The right of Figure 6 shows numerical convergence in time discretization. It should be noted that both TR-only and $\mathrm{T}+\mathrm{M}$ take the staggered approach. We take a solution of $\mathrm{T}+\mathrm{M}$ from tiny time step size as a reference solution. From the convergence analysis, we find first order accuracy in time for the staggered $\mathrm{T}+\mathrm{M}$. Thus, the sequential algorithm proposed in this paper can increase accuracy by reducing time step size. It should be noted that accuracy cannot be improved even though time step size is reduced, when we use other obvious sequential methods in coupled flow and geomechanics (e.g., non-convergence of the drained method and the fixed-strain method) (Kim et al., 2011a,b).

\subsubsection{D elastic chemo-thermo-poro-mechanics}

We further test a 1D elastic non-isothermal chemo-thermo-poro-mechanics, using the same domain, initial and boundary conditions, and the input data as those used in the previous 1D chemo-poro-mechanics problem. Initial temperature is $T_{i}=240{ }^{\circ} \mathrm{C}$, and the monitoring point is located at the gridblock of the right end, where liquid water at $20^{\circ} \mathrm{C}$ is injected at a total mass rate of $Q_{i n j}=1.0 \times 10^{-2} \mathrm{~kg} \cdot \mathrm{s}^{-1}$. We have a specific heat capacity of $1000 \mathrm{~J} \mathrm{~kg}^{-1}{ }^{o} \mathrm{C}^{-1}$, a thermal dilation coefficient of $\alpha_{T}=4.5 \times 10^{-5}{ }^{\circ} \mathrm{C}^{-1}$, and a thermal conductivity of $k_{T}=3.1 \mathrm{Wm}^{-1 o} \mathrm{C}^{-1}$. In this $1 \mathrm{D}$ case, as aforementioned, TR-only can solve the coupled flow and geomechanics problem by using the exact pore-compressibility and thermal expansivity in flow, which are $0.9804 \times 10^{-9} \mathrm{~Pa}^{-1}$ and $3.1765 \times 10^{-4}{ }^{o} C^{-1}$, respectively. We inject liquid water that has zero specific enthalpy, which corresponds to water at $0^{\circ} \mathrm{C}$, in order to investigate noticeable thermal behavior.

Figure 8 shows comparison of pressure and temperature between 'TR-only' and ' $\mathrm{T}+\mathrm{M}$ ' as well as convergence study of $\mathrm{T}+\mathrm{M}$ in time discretization. The results of $\mathrm{T}+\mathrm{M}$ match those of TR-only. $\mathrm{T}+\mathrm{M}$ shows first-order accuracy in time discretization when time step size is refined, same as the previous 1D elastic chemo-poro-mechanics test. This implies that the proposed sequential method does not lose accuracy of the backward Euler method, which is first-order in time. From the fast convergence of the full iterative approach and the first order accuracy of the staggered approach, the proposed algorithm is comparable to the monolithic (fully-coupled) method. 


\subsection{D elastic chemo-thermo-poro-mechanics}

We consider a naturally fractured geothermal problem shown in the left of Figure 9. The geothermal reservoir has a large dimension perpendicular to the fracture plane, i.e., the problem can be reduced to one of multiple porosity 2D plane strain geomechanics, as shown in the right of Figure 9. For the $2 \mathrm{D}$ problem, we have $20 \times 10$ gridblocks in the $\mathrm{x}$ and $\mathrm{z}$ directions, the sizes of which are uniform, $10.0 \mathrm{~m}$. We assume the size of the dimension in the $\mathrm{y}$ direction to be $500 \mathrm{~m}$ in flow, large enough for the constant temperature boundary for heat flow. Each gridblock has five subelements, one fracture and four rock matrix media, the volume fractions of which are $0.002,0.098,0.20,0.35$, and 0.35 , respectively.

The reservoir is fully water saturated, and the initial pressure and temperature at the top are $19.0 \mathrm{MPa}$ and $146.7^{\circ} \mathrm{C}$, respectively, which might be much cooler than a producing geothermal system, but which shows qualitatively the same behavior. They are distributed with the hydrostatic pressure gradient, $9.806 \mathrm{kPa} / \mathrm{m}$, and the geothermal gradient, $25^{\circ} \mathrm{C} / \mathrm{km}$. We impose no-flow boundaries of heat and fluid on all sides, assuming an alternative injection and production scenario with symmetry. The monitoring points are the injection and production points at the fracture medium (P1 and P2, respectively), as shown in the left of Figure 9. For geomechanics, the initial vertical total stress is -19.0 MPa at the top, and vertically distributed based on the bulk density, $2200 \mathrm{~kg} / \mathrm{m}^{3}$. The horizontal total stress in the $\mathrm{x}$ direction is the same as the vertical stress. The total stress in the y direction is then determined based on the plane strain condition with given geomechanical moduli described below. We have 19.0 MPa overburden, and horizontally constrained boundaries at the sides and no vertical displacement at the bottom. Other flow and geomechanical properties are listed in Table 1.

For geochemistry, we take the same quartz dissolution process used in the previous 1D chemo-poro-mechanics problem. We have the initial $C_{S i}$ obtained from the chemical equilibrium at initial time, taking $C_{S i, q z, 0}=2.078 \times$ $10^{-3} \mathrm{~mol} / \mathrm{kg}$ at $147^{\circ} \mathrm{C}$, because we found from the previous $1 \mathrm{D}$ chemo-poro-mechanic problem that non-equilibrium at initial time changed pressure and concentration noticeably. It should be noted that $C_{S i, q z}$ is a function of temperature, from which $C_{S i, q z}$ varies during the simulation as temperature changes.

We use dynamic permeability and Young's modulus hereafter, assuming that deformation of fractured medium is small. The assumption of small deformation is consistent with the infinitesimal transformation, which allows us to use Equation 2. Then, we employ the strain and failure-dependent permeability and the strain-dependent Young's modulus, 
motivated by the Cubic law (Snow, 1965; Witherspoon et al., 1980) and the relation between the normal joint stiffness and the normal closure (Bandis et al., 1983), respectively, as follows.

$$
k_{p, i i}=k_{p, i i, 0} \lambda^{f}\left(1+\varepsilon_{i i}\right)^{3}, i i=x x, y y, z z
$$

where the subscript $(\cdot)_{0}$ indicates the reference state. $\lambda^{f}$ is the permeability multiplier, determined from the failure status. In other words, a high value is assigned dynamically, when a porous medium faces failure. If the material faces shear failure, $\lambda^{f}$ becomes $100 \times N_{f g} / N_{g}$ in this study, where $N_{g}$ and $N_{f g}$ are the numbers of total and failed Gauss points with a gridblock, respectively. $\lambda^{f}$ is constant for the $2 \mathrm{D}$ elastic problem of this subsection, while it will vary for the 3D elastoplastic problem in the next subsection.

$$
E_{i}=E_{i, 0} \frac{1}{\left(1+\varepsilon_{i i}\right)^{2}}, i=x, y, z,
$$

where $E$ is Young's modulus. Equations 19 and 20 are examples of nonlinear permeability and geomechanical moduli. It should be noted that one may use different types of the nonlinear permeability and Young's modulus depending on specific reservoir conditions.

Figure 10 shows the comparison between chemo-thermo-poro-mechanics (with reactive transport) and thermoporo-mechanics only (without reactive transport). In Figure 10 (a), for both results, we observe an increase in pressure at early time, resulting from fluid injection as well as thermal contraction. Figures 10 (b) and (c) show that, as temperature decreases, volumetric strain $\varepsilon_{v}$ decreases, inducing compaction of the reservoir, although fluid is continuously injected. Both volumetric strains with and without reactive transport still decrease even after there is no further decrease of temperature at $\mathrm{P} 1$, because compaction at other areas induces the immediate compaction at $\mathrm{P} 1$. This compaction results in the additional increases of pressure at P1, as shown in Figure 10 (a). Then, because of high permeability, pressure at P2 also increases due to the increased pressure at P1, showing the same trend (Figure 10 (d)). Note that the perturbation of geomechanics at a certain location affects the entire domain instantaneously, because of the nature of quasi-static mechanics. These nonlocal responses, which originate in different time scales between flow and geomechanics, cannot properly be captured by a flow-only simulation, but only by the coupled flow-geomechanics simulation.

The comparison between the simulation results with and without reactive transport in Figure 10 (a) shows that pressure with reactive transport is lower than that without reactive transport, because dissolution of quartz results in 
an increase of pore-volume, followed by a pressure drop, just as discussed in the previous 1D chemo-poro-mechanics problem. The difference in pressure at P1 causes differences in compaction (Figure $10(\mathrm{~b})$ ) and pressure at P2 (Figure 10 (d))

Figure 11 shows the evolutions of $C_{S i}$ and the dissolutions of quartz at different locations of the fracture medium (Figures 11 (a) and (b), respectively), and those at different porous media of the gridblock that contains the injection point (Figures 11 (c) and (d)). In Figure 11 (a), $C_{S i}$ is the lowest at the injection point, $x_{d}=0.975(\mathrm{x}=195 \mathrm{~m})$ of the fracture medium, because $C_{S i}$ of injected water is zero. Interestingly, as shown in Figure 11 (b), dissolution of quartz is higher at $x_{d}=0.775(\mathrm{x}=155 \mathrm{~m})$ of the fracture medium than at the injection point. Higher temperature at $x_{d}=0.775$ of the fracture medium than that at the injection point yields higher $C_{S i, q z}$ and higher $\kappa_{q z}$, which in turn lead to higher dissolution rate of quartz. In Figures 11 (c) and (d), $C_{S i}$ within the fracture medium is the lowest and dissolution of quartz is the highest within the fracture medium among all the continua of the gridblock, because permeabilities of the rock matrix media are extremely low, and liquid water mainly flows through the fracture medium, not intruding into the rock matrix media.

When comparing the coupled flow-geomechanics simulation with the flow-only simulation, we find from Figures 10 (a) and 12 significant differences, for example, in pressure and porosity. In the left of Figure 12, TR-only with reactive transport shows more pressure drop than TR-only without reactive transport, because the dissolution increases pore volume, which causes the pressure to drop below the initial pressure, just as shown in the left of Figure 6 . On the other hand, we observe that the pressure of chemo-thermo-poro-mechanics is higher than that from TR-only with reactive transport, because the pressure build-up by thermal contraction over the domain completely offsets the pressure drop from the change in pore volume induced by the quartz dissolution.

\subsection{D elastoplastic chemo-thermo-poro-mechanics}

We introduce an artificially fracturing geothermal problem. Figure 13 shows a 3D domain, which has an initial elliptic fracture at the center. We take, by symmetry, the upper and right quadrant as a simulation domain. For the 3D problem, we have $15 \times 15 \times 19$ gridblocks in the $\mathrm{x}, \mathrm{y}$, and $\mathrm{z}$ directions, employing the single porosity model. The sizes of gridblocks in the $\mathrm{x}$ and $\mathrm{y}$ directions are uniform, $1.0 \mathrm{~m}$, while those in the $\mathrm{z}$ direction are not uniform; $1.0 \mathrm{~m}$ from the first gridblock to the $8^{\text {th }}$ in depth, $0.2 \mathrm{~m}, 0.04 \mathrm{~m}, 0.01 \mathrm{~m}, 0.04 \mathrm{~m}, 0.2 \mathrm{~m}$ from the $9^{\text {th }}$ to the $13^{\text {th }}$, respectively, and $1.0 \mathrm{~m}$ for the rest gridblocks. 
The reservoir is fully water-saturated, and the initial pressure and temperature at the top are $15.1 \mathrm{MPa}$ and $146.7^{\circ} C$, respectively. They are distributed with the hydrostatic pressure gradient, $9.806 \mathrm{kPa} / \mathrm{m}$, and the geothermal gradient, $25^{\circ} \mathrm{C} / \mathrm{km}$. The initial permeabilities of the fracture and the rock matrix are $5.9 \times 10^{-13} \mathrm{~m}^{2}(0.6$ Darcy) and $5.9 \times$ $10^{-17} \mathrm{~m}^{2}(0.06 \mathrm{mD})$, respectively, where we assume the rock matrix to exhibit some microcracks, having higher permeability of the rock matrix than that at the previous 2D problem. We impose no-flow boundaries on all sides. The injection well is located at $\mathrm{x}=0 \mathrm{~m}$ and $\mathrm{y}=0 \mathrm{~m}$ at the $10^{\text {th }}$ layer of the $\mathrm{z}$ direction (Figure 13). The injection rate is $0.25 \mathrm{~kg} / \mathrm{s}$ with $146.7^{\circ} \mathrm{C}$ water, assuming water to become heated at the bottom hole. For geomechanics, the initial vertical total stress is $-21.58 \mathrm{MPa}$ at the top, vertically distributed based on the bulk density, $2200 \mathrm{~kg} / \mathrm{m}^{3}$. The horizontal total stresses in the $\mathrm{x}$ and $\mathrm{y}$ directions are both $-23.74 \mathrm{MPa}$ at the top and vertically distributed with the gradient of $-23.73 \mathrm{kPa} / \mathrm{m}$, which become 1.1 times the vertical total stress. We have $21.58 \mathrm{MPa}$ overburden and no vertical displacement at the bottom. We assign low Young's modulus to the last $\left(15^{\text {th }}\right)$ horizontal layer in the $\mathrm{x}$ and $\mathrm{y}$ directions to maintain the initial horizontal stress at the sides. Other input data including geochemistry are the same as those in the previous $2 \mathrm{D}$ chemo-thermo-poro-mechanics problem.

For the modeling of plasticity, we consider shear failure from the Mohr-Coulomb model, having $c_{h}=10.0 \mathrm{MPa}$, and $\Psi_{f}=\Psi_{d}=\pi / 18$, where $\Psi_{f}$ and $\Psi_{d}$ are the friction and dilation angles, respectively, and $c_{h}$ is the cohesion. This failure implicitly aims at application of hydro-shearing for the situations where shear failure occurs, activating micro-cracks and stimulating the reservoir.

Once a material faces failure, entering plasticity, a substantial permeability change is applied to simulate the transition from intact rock permeability to that of a fractured rock permeability. Specifically, we dynamically modify the reference permeability of the rock matrix, $5.9 \times 10^{-17} \mathrm{~m}^{2}$, into $5.9 \times 10^{-15} \mathrm{~m}^{2}$ (i.e., $\lambda_{i i}^{f}=10^{2}$ in Equation 19). We have a monitoring point, $\mathrm{P} 1\left(x_{d}=0.5(\mathrm{x}=7.5 \mathrm{~m}), y_{d}=0.033(\mathrm{y}=0.5 \mathrm{~m})\right)$, shown in Figure 13.

Figure 14 shows comparison of fractured areas with and without reactive transport at the same time, $t_{d}=9.76$ $(\mathrm{t}=12.1 \mathrm{~min})$. From the comparison, we find differences of the failed areas between the two cases. For the case with reactive transport, we find more extensive horizontal and vertical failures, compared with the case without reactive transport. However, this simulation result does not always mean that simulation with reactive transport cause larger fracturing than simulation without reactive transport, but their differences depend on various reservoir conditions and injection scenarios. 
Because of this substantial increase of permeability induced by material failure, we find from Figure 15 that the pressures with and without reactive transport are different at late times. For the case with reactive transport, depressurization due to fast growth of high permeable fractured areas, shown in Figure 14 and Figure 15 (right), seems more dominant than pressurization from injection.

From Figure 16, $C_{S i}^{d}$ at the injection point decreases considerably from initial time because zero $C_{S i}^{d}$ of water is injected, while $C_{S i}^{d}$ 's at other points decrease at a slower rate. Noticeable dissolution of quartz is found as zero $C_{S i}$ of water is injected.

\section{Conclusions}

We proposed a sequential implicit formulation and algorithm for chemo-thermo-poro-mechanics, and implemented the coupling algorithm within a coupled flow-geomechanics simulator. This simulator can handle the multiple porosity model in fractured geothermal reservoirs, and can consider nonlinear permeability and geomechanics, including plasticity. We validated the coupled geomechanics-flow-reactive transport simulator, using the analytical solutions for the poromechanical effects and the reference solutions that can be computed for the $1 \mathrm{D}$ elastic cases. We tested the behavior of numerical convergence for the proposed sequential algorithm, and it yielded fast convergence and kept first-order accuracy in time, comparable to the monolithic approach.

From numerical simulation of the naturally 2D fractured geothermal reservoir with the multiple porosity model, we demonstrated how chemo-thermo-poro-mechanics can yield significantly different results from thermo-poro-mechanics without reactive transport, because small pore volume changes can lead to large fluid pressure effects for chemically sensitive reservoirs in which incompressible fluid is saturated. From the 3D shear fracturing problem induced by fluid injection, we found the differences in failure evolution, causing substantial changes in permeability, for the cases with and without reactive transport. The changes in pore volume, permeability, and fluid pressure caused different evolutions of plastic failure. Thus, we recommend employing an integrated chemo-thermo-poro-mechanics simulator for chemically and thermally sensitive and highly deformable reservoirs such as fractured geothermal reservoirs. The proposed sequential algorithm enables straightforward construction of the interface between existing flow-reactive transport and geomechanics simulators. 


\section{Acknowledgements}

This work was supported by the American Recovery and Reinvestment Act (ARRA), through the Assistant Secretary for Energy Efficiency and Renewable Energy (EERE), Office of Technology Development, Geothermal Technologies Program, of the U.S. Department of Energy under Contract No. DE-AC02-05CH11231, and by 2011 TOUGH2 development grants in the Earth Sciences Division of Lawrence Berkeley National Laboratory.

\section{References}

Armero F. 1999. Formulation and finite element implementation of a multiplicative model of coupled poro-plasticity at finite strains under fully saturated conditions. Computer Methods in Applied Mechanics and Engineering 171: 205-241.

Aziz K. and Settari A. 1979. Petroleum Reservoir Simulation. London: Elsevier.

Bai M. 1999. On equivalence of dual-porosity poroelastic parameters. Journal of Geophysical Research 104: 10,461 $-10,466$.

Bandis S., Lumsden A., and Barton N. 1983. Fundamentals of rock joint deformation. International Journal of Rock Mechanics and Mining Sciences and Geomechanics Abstracts 20: 249-268.

Barenblatt G.E., Zheltov I.P., and Kochina I.N. 1960. Basic concepts in the theory of seepage of homogeneous liquids in fissured rocks. Journal of Applied Mathematics 24(5): 1286 - 1303.

Berryman J.G. 2002. Extension of poroelastic analysis to double-porosity materials: New technique in microgeomechanics. Journal of Engineering Mechanics-ASCE 128(8): 840 - 847.

Biot M.A. 1941. General theory of three-dimensional consolidation. Journal of Applied Physics 12: 155-164.

Biot M.A. and Willis D.G. 1957. The elastic coefficients of the theory of consolidation. Journal of Applied Physics 594-601.

Coussy O. 1995. Mechanics of porous continua. Chichester, England: John Wiley and Sons.

Geertsma J. 1957. The effect of fluid pressure decline on volumetric change of porous rocks. Petroleum Transactions, AIME 210: 331-340. 
Johnson J.W., Knauss K.G., Glassley W.E., DeLoach L.D., and Tompson A.F.B. 1998. Reactive transport modeling of plug-flow reactor experiments: quartz and tuff dissolution at $240{ }^{\circ} \mathrm{C}$. Journal of Hydrology 209: 81-111.

Kim J. and Moridis G.J. 2014. Gas flow tightly coupled to elastoplastic geomechanics for tight and shale gas reservoirs: material failure and enhanced permeability. SPE Journal SPE 155640, http://dx.doi.org/10.2118/155640-PA.

Kim J., Sonnenthal E., and Rutqvist J. 2012. Formulation and sequential numerical algorithms of coupled fluid/heat flow and geomechanics for multiple porosity materials. International Journal for Numerical Methods in Engineering 92: $425-456$.

Kim J., Tchelepi H.A., and Juanes R. 2011a. Stability and convergence of sequential methods for coupled flow and geomechanics: Drained and undrained splits. Computer Methods in Applied Mechanics and Engineering 200: 2094-2116.

Kim J., Tchelepi H.A., and Juanes R. 2011b. Stability and convergence of sequential methods for coupled flow and geomechanics: Fixed-stress and fixed-strain splits. Computer Methods in Applied Mechanics and Engineering 200: 1591-1606.

Kim J., Tchelepi H.A., and Juanes R. 2013. Rigorous coupling of geomechanics and multiphase flow with strong capillarity. SPE Journal 18(6): 1591-1606. Spe 141268.

Klinkenberg L.J. 1941. The permeability of porous media to liquid and gases. API Drilling and Production Practice, $200-213$

Knauss K.G. and Copenhaver S.A. 1995. The effect of malonate on the dissolution kinetics of albite, quartz and microcline as a function of $\mathrm{pH}$ at $70{ }^{\circ} \mathrm{C}$. Applied Geochemistry 10(1): 14-33.

Lewis R.W. and Schrefler B.A. 1998. The finite element method in the static and dynamic deformation and consolidation of porous media. Chichester, England: Wiley, 2nd edition.

Moridis G.J., Kowalsky M.B., and Pruess K. 2008. TOUGH+HYDRATE v1.0 User's Manual: A Code for the Simulation of System Behavior in Hydrate-Bearing Geologic Media. Report LBNL-00149E, Lawrence Berkeley National Laboratory, Berkeley, CA. 
Phillips P.J. and Wheeler M.F. 2007. A coupling of mixed and continuous Galerkin finite element methods for poroelasticity I: the continuous in time case. Computational Geosciences 11: 131-144.

Pruess K. and Narasimhan T.N. 1985. A practical method for modeling fluid and heat flow in fractured porous media. SPE Journal 25(1): $14-26$.

Pruess K., Oldenburg C., and Moridis G. 1999. TOUGH2 User's Guide, Version 2.0. Report LBNL-43134, Lawrence Berkeley National Laboratory, Berkeley, CA.

Rutqvist J. and Stephansson O. 2003. The role of hydromechanical coupling in fractured rock engineering. Hydrogeology Journal 11: 7-40.

Settari A. and Mourits F. 1998. A coupled reservoir and geomechanical simulation system. SPE Journal 3: 219-226.

Snow D.T. 1965. A parallel plate model of fractured permeable media. Ph.D. Dissertation, University of California, Berkeley.

Sonnenthal E., Ito A., Spycher N., Yui M., Apps J., Sugita Y., Conrad M., and Kawakami S. 2005. Approaches to modeling coupled thermal, hydrological, and chemical processes in the Drift Scale Heater Test at Yucca Mountain. International Journal of Rock Mechanics and Mining Sciences 42: 698-719.

Taron J., Elsworth D., and Min K. 2009. Numerical simulation of thermo-hydrologic-mechanical-chemical processes in deformable, fractured porous media. International Journal of Rock Mechanics and Mining Sciences 46: 842 854.

Tester J.W., Worley W., Robinson B.A., Grigsby C.O., and Feerer J.R. 1994. Correlating quartz dissolution kinetics in pure water from 25 to $625^{\circ} \mathrm{C}$. Geochimica et Cosmochimica Acta 58(11): 2407-2420.

Vermeer P.A. and Verruijt A. 1981. An accuracy condition for consolidation by finite elements. International Journal for Numerical and Analytical Methods in Geomechanics 5: 1-14.

Witherspoon P.A., Wang J.S.Y., Iwai K., and Gale J.E. 1980. Validity of Cubic Law for fluid flow in a deformable rock fracture. Water Resources Research 16(6): 1016-1024. 
Xu T., Sonnenthal E., Spycher N., and Pruess K. 2006. TOUGHREACT: A simulation program for non-isothermal multiphase reactive geochemical transport in variably saturated geologic media: Applications to geothermal injectivity and $\mathrm{CO} 2$ geological sequestration. Computers and Geosciences 32: 145-156.

Xu T., Sonnenthal E., Spycher N., and Pruess K. 2008. TOUGHREACT User's guide: A simulation program for nonisothermal multiphase reactive geochemical transport in variably saturated geologic media. LBNL-55460-2008, Lawrence Berkeley National Laboratory, Berkeley, CA.

Xu T., Spycher N., Sonnenthal E., Zhang G., Zheng L., and Pruess K. 2011. TOUGHREACT Version 2.0: A simulator for subsurface reactive transport under non-isothermal multiphase flow conditions. Computers and Geosciences 37 : $763-774$
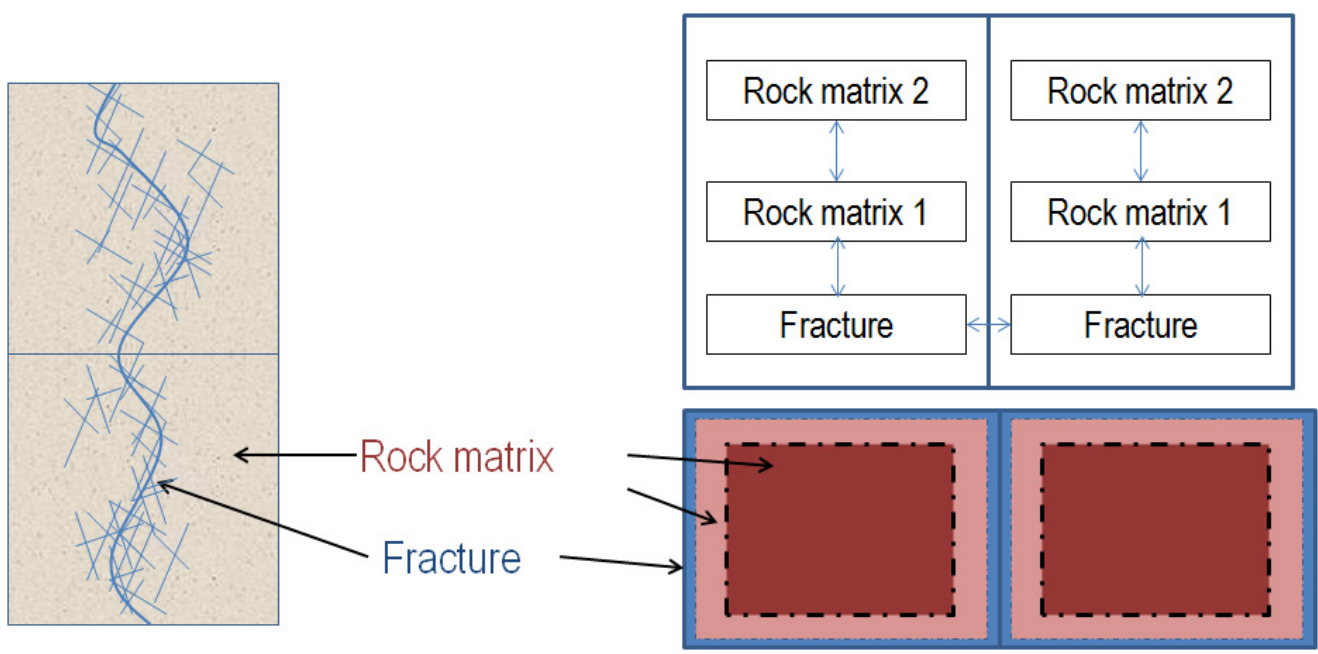

Figure 1: Left: a schematic diagram of a fracture-matrix system. Right: a conceptual model of the multiple interacting continuum (MINC) model (Pruess and Narasimhan, 1985). In the MINC model, fluid flows though the fracture medium over the domain. On the other hand, the rock matrix medium stores fluid and conveys it to the fracture medium. We may have several rock matrix media for accurate modeling of fluid-heat flow. 


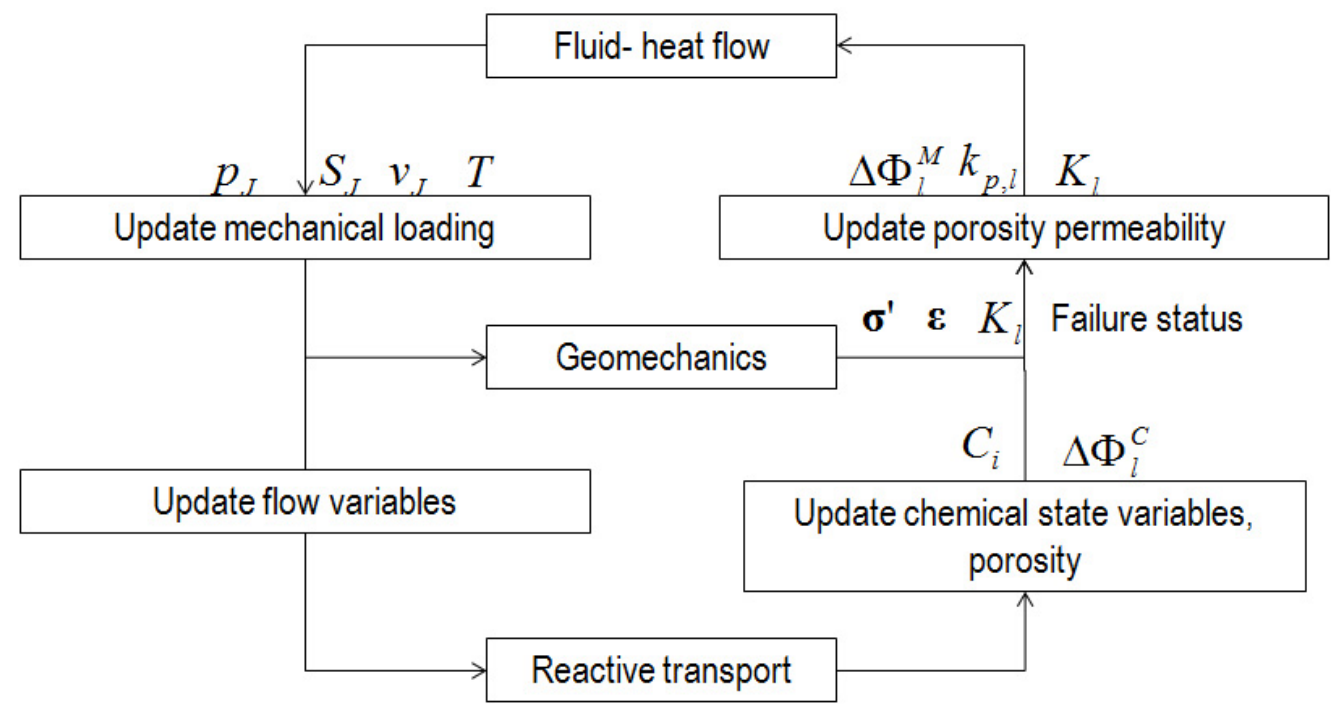

Figure 2: The sequential implicit algorithm of chemo-thermo-poro-mechanics. Flow, geomechanics, reactive transport simulators are communicated sequentially.

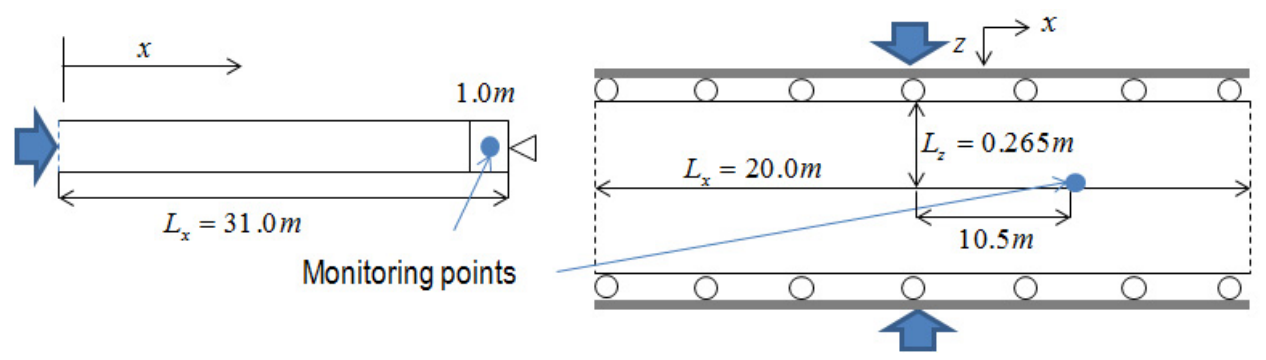

Figure 3: Left: Terzaghi's problem. Right: Mandel's problem. Verification for poromechanical effects is tested. 

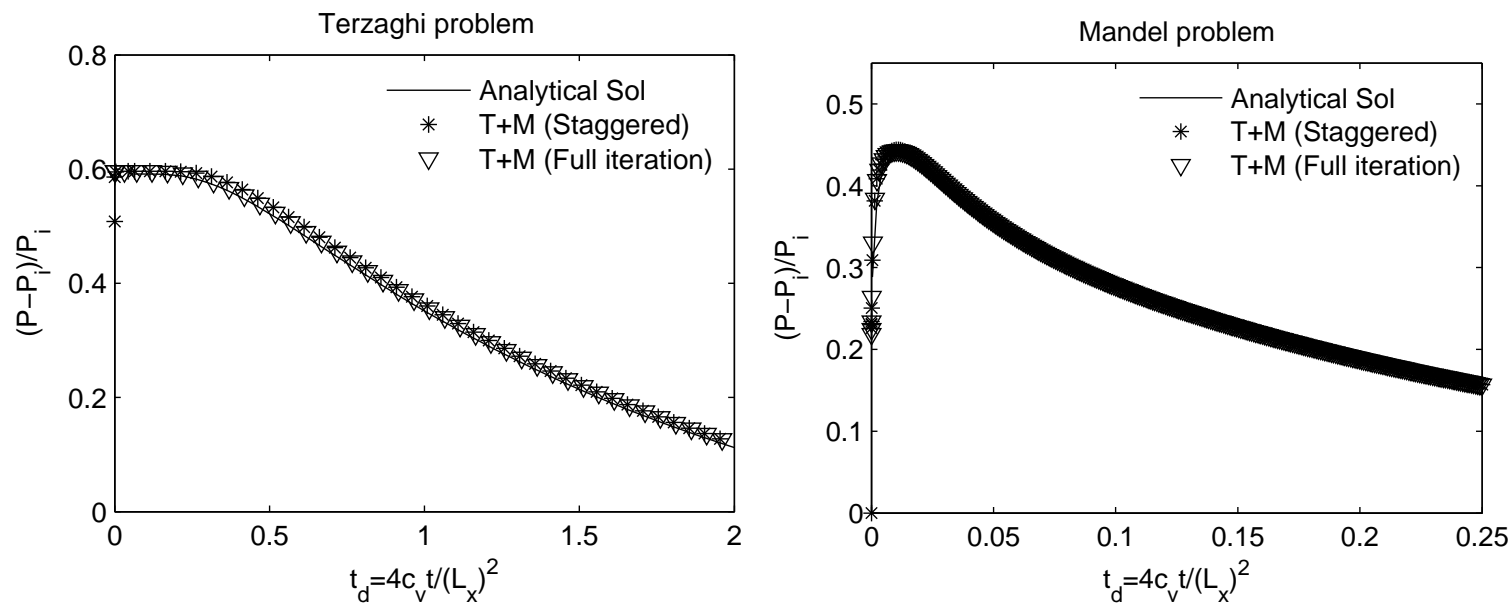

Figure 4: Comparison between numerical solutions of T+M and analytical solutions of Terzaghi's problem (left) and Mandel's problem (right). $\mathbf{T}+\mathbf{M}$ matches the analytical solutions. $c_{v}$ is the consolidation coefficient, defined as $c_{v}=\frac{k_{p}}{\mu_{w}\left(1 / K_{d r}+\phi c_{w}\right)} \cdot t$ is time. $P_{i}$ is the initial reservoir pressure.
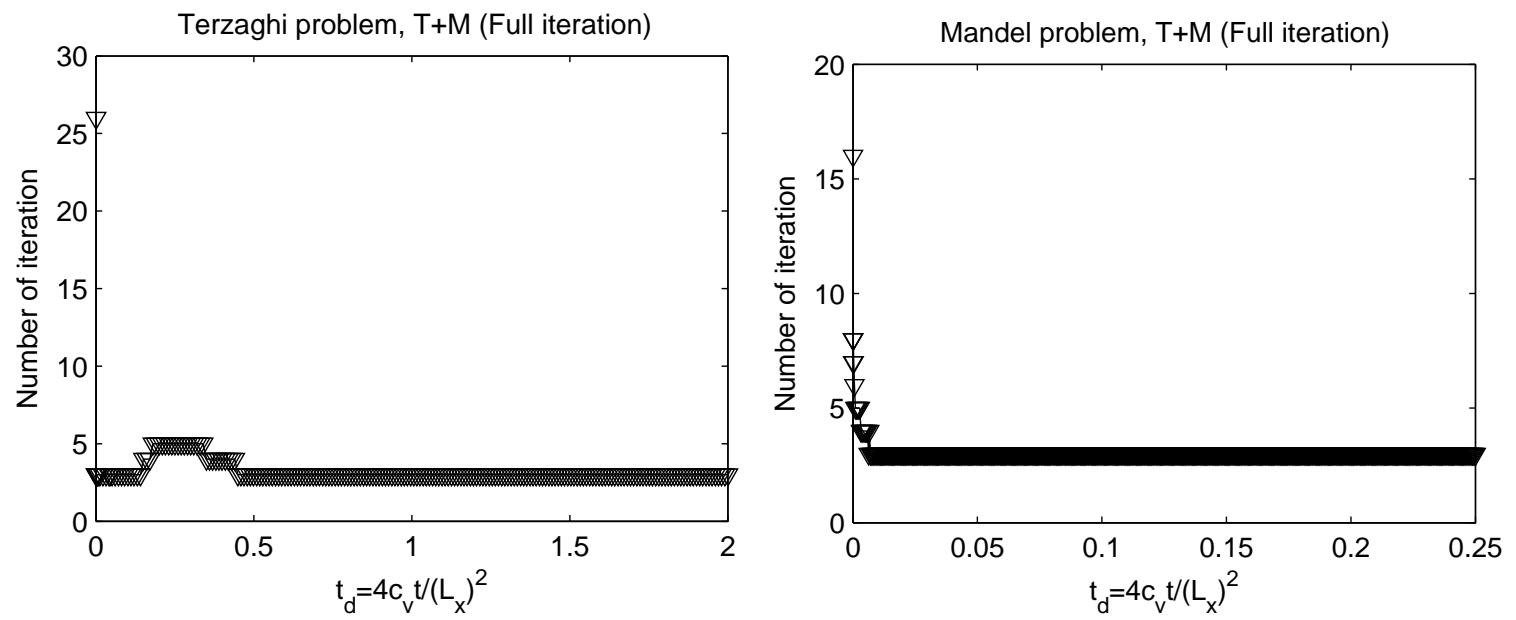

Figure 5: Number of iterations required for convergence with a prescribed tolerance during simulation for $\mathbf{T}+\mathbf{M}$ when full iteration is performed. The fixed-stress split almost takes 3 iterations for Terzaghi's and Mandel's problems, which shows fast convergence when it takes full iteration. The tolerance of convergence is $\left|\frac{P^{j}-P^{j-1}}{P_{i}}\right|_{x=x_{0}}<10^{-5}$, where the superscript $j$ indicates the iteration level. $x_{0}$ is the monitoring point. 

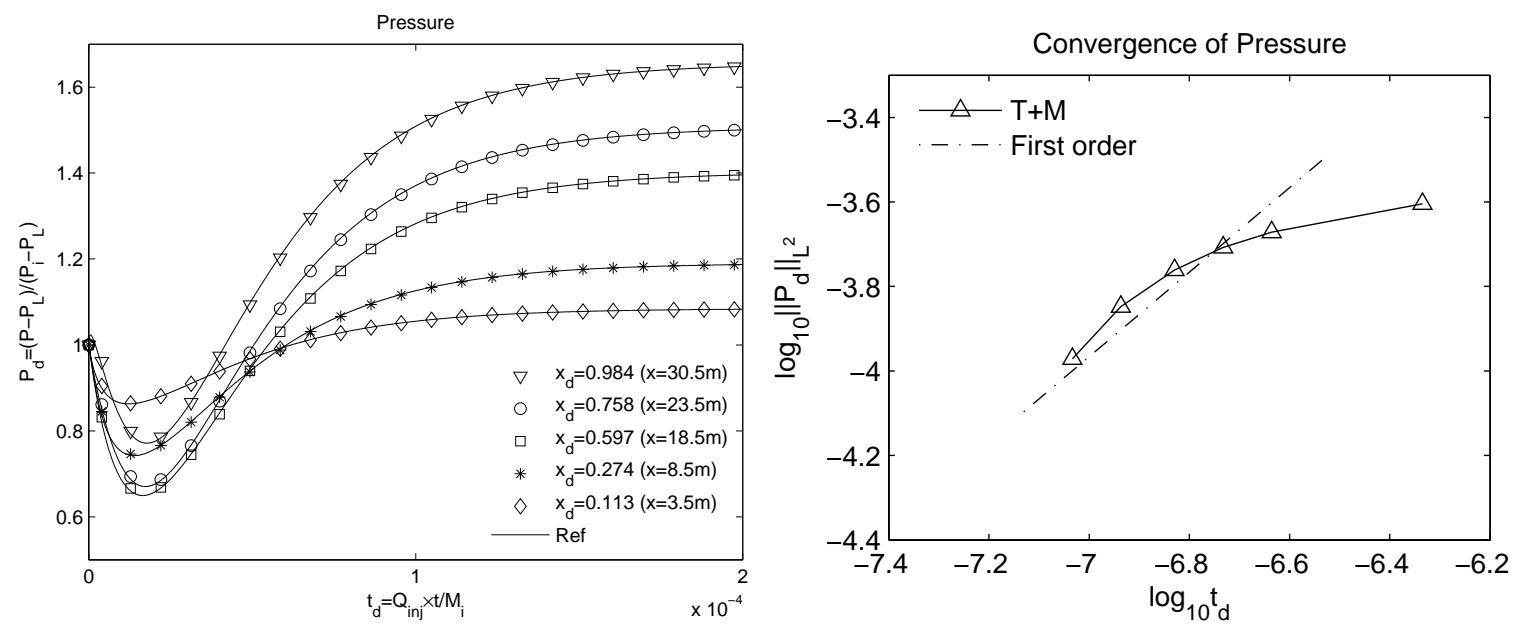

Figure 6: Left: Evolution of pressure and Comparison of pressure between TR-only and T+M for the 1D elastic chemo-poromechanics problem. The results from $\mathrm{T}+\mathrm{M}$ match the results from TR-only. Right: Convergence analysis in time discretization. $\mathbf{T}+\mathbf{M}$ shows first-order accuracy in time. $M_{i}$ is the fluid mass in place at initial time, $1.08 \times 10^{4} \mathrm{~kg} . P_{L}=8.2 \mathrm{MPa}$.
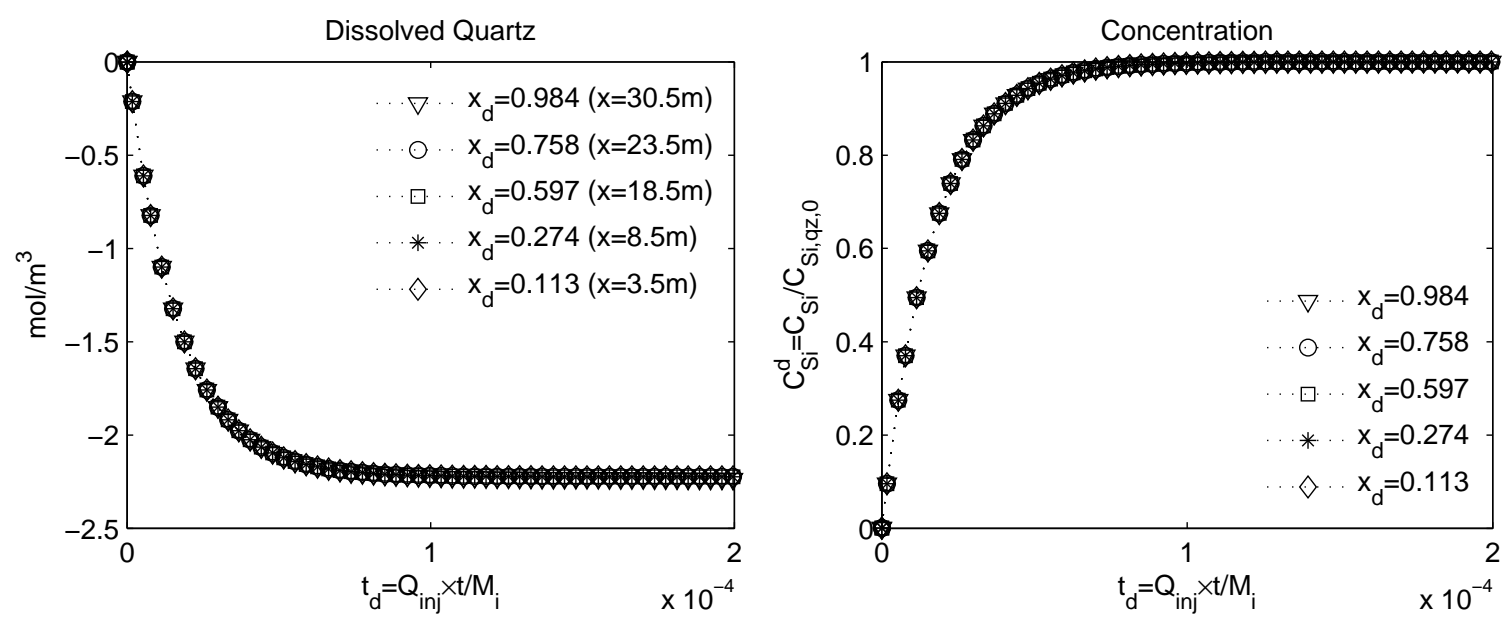

Figure 7: Left: dissolution of quartz at different monitoring points. Right: evolution of $C_{S i}$ at different monitoring points. $x_{d}=x / L_{x}$ is the dimensionless distance, and $C_{S i}^{d}$ is the normalized concentration. As quartz is dissolved into water, $C_{S i}$ increases. 

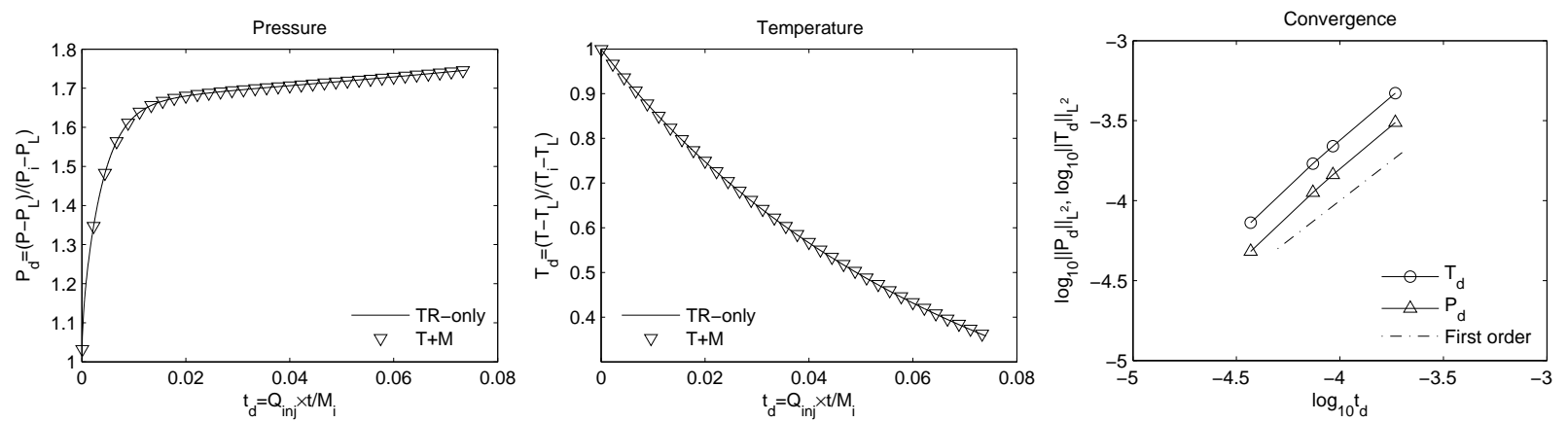

Figure 8: Comparison of (a) pressure and (b) temperature between TR-only and T+M for the 1D elastic chemo-thermo-poromechanics problem. The results from $T+M$ match the results from TR-only. (c) Convergence analysis in time discretization. $\mathbf{T}+\mathbf{M}$ shows first-order accuracy in time. $P_{L}=0.0 \mathrm{~Pa}$, and $T_{L}=0.0{ }^{\circ} \mathrm{C}$.
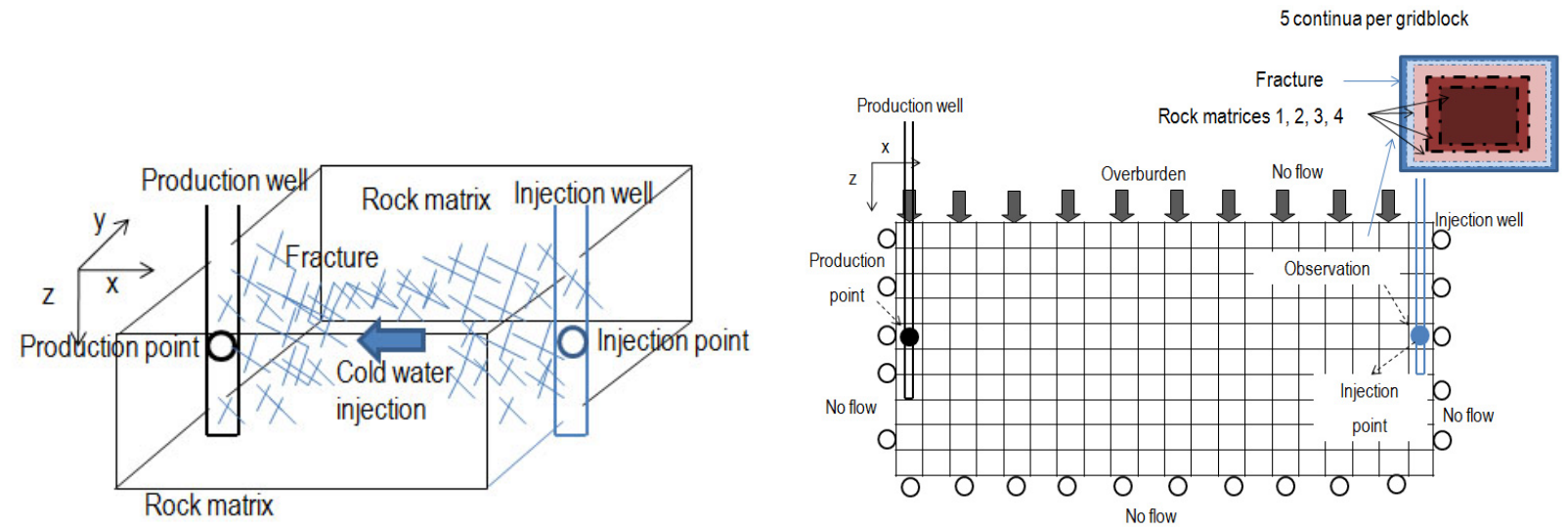

Figure 9: Left: the schematics of a 3D fractured geothermal reservoir. Right: the discretized domain of the fractured geothermal reservoir with the 2D plane strain geomechanics and the multiple porosity model.

Table 1: Input data for flow and geomechanics

\begin{tabular}{|c|c|c|}
\hline Permeability & $5.9 \times 10^{-13} \mathrm{~m}^{2}$ (fracture) & $5.9 \times 10^{-19} \mathrm{~m}^{2}$ (rock matrix) \\
\hline Porosity & 0.5 (fracture) & 0.3 (rock matrix) \\
\hline Heat conductivity & $0.5 \mathrm{Wm}^{-1 o} \mathrm{C}^{-1}$ (desaturated), & $3.1 W m^{-1 o} C^{-1}$ (saturated) \\
\hline Specific heat capacity & $1000 \mathrm{~J} \mathrm{~kg}^{-1{ }^{o}} \mathrm{C}^{-1}$ (all) & \\
\hline Injection \& Production & $50.0 \mathrm{~kg} / \mathrm{s}$ at $20^{\circ} \mathrm{C}$, & $50.0 \mathrm{~kg} / \mathrm{s}$ \\
\hline Young's modulus & 213.75 MPa (fracture), & 2.234 GPa (rock matrix) \\
\hline Poisson's ratio & 0.0 (fracture), & 0.3 (rock matrix) \\
\hline Biot's coefficient & 1.0 (all) & \\
\hline Thermal dilation coefficient & $\alpha_{T}=4.5 \times 10^{-5 o} C^{-1}$ (all) & \\
\hline
\end{tabular}


(a) P1

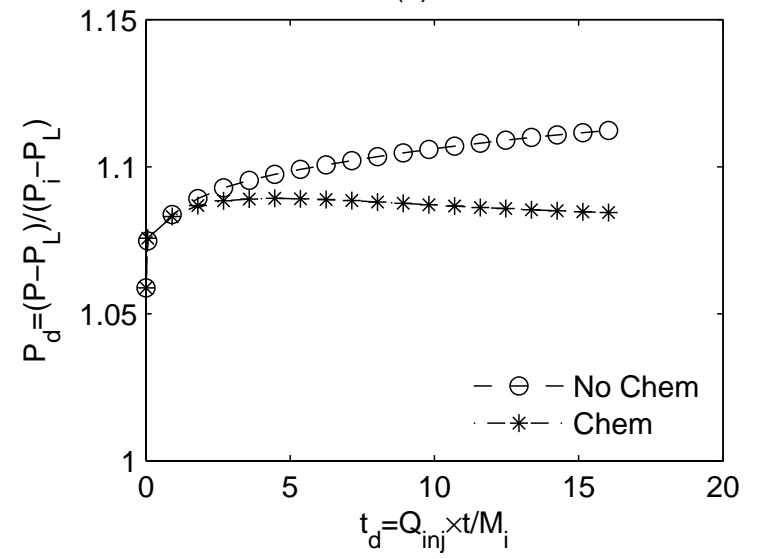

(c) P1

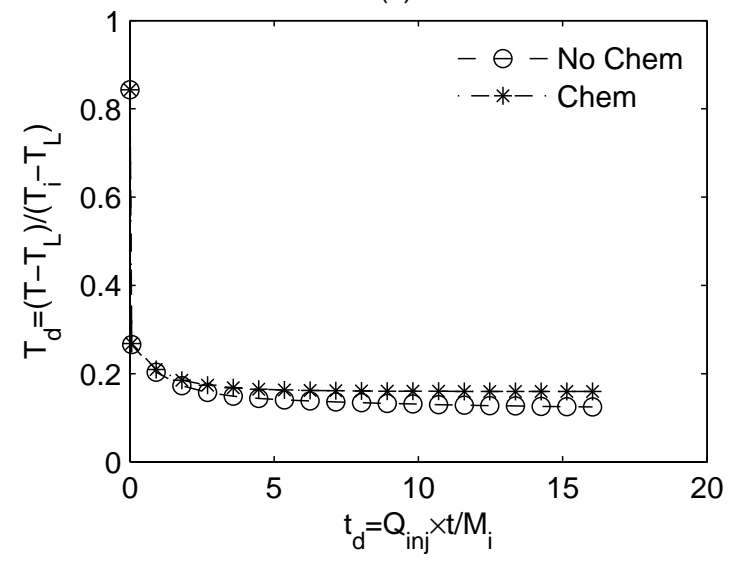

(b) P1

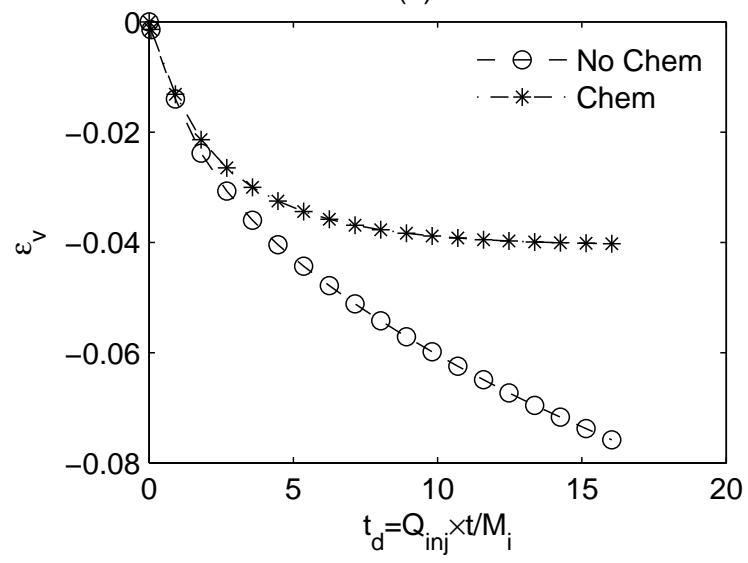

(d) P2

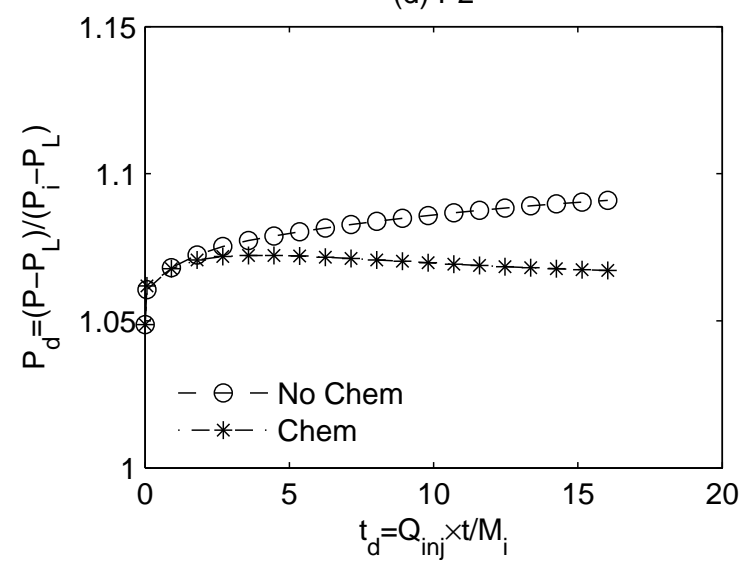

Figure 10: Comparison of the results between chemo-thermo-poro-mechanics, ('Chem'), and thermo-poro-mechanics only, ('No chem'). (a) pressure at $\mathbf{P 1}$, the injection point at the fracture medium. (b) $\varepsilon_{v}$ at $\mathbf{P} 1$. (c) temperature at $\mathbf{P 1}$. (d) pressure at $\mathbf{P} 2$, the production point at the fracture medium. $M_{i}$ is the initial fluid mass in place at the fracture medium, $1.304 \times 10^{7} \mathrm{~kg}$. $P_{L}=10 \mathrm{MPa}$ and $T_{L}=0.0^{\circ} \mathrm{C}$. 
(a) Fracture at $z_{d}=0.45(z=45 m)$

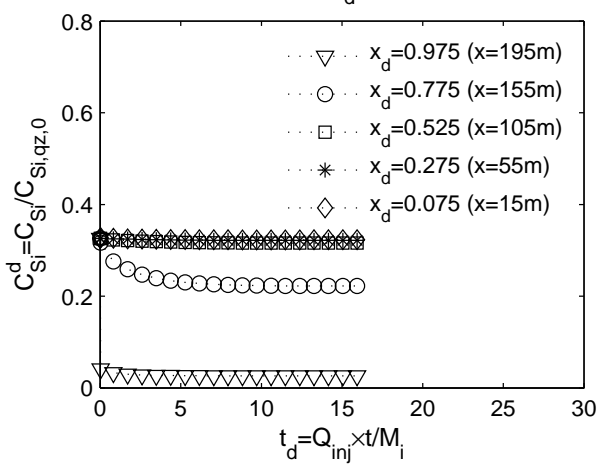

(c) At $x_{d}=0.975, z_{d}=0.45(z=45 m)$

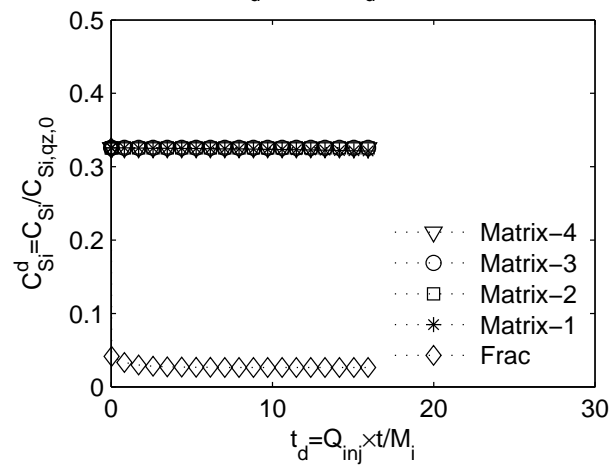

(b) Fracture at $z_{\mathrm{d}}=0.45(\mathrm{z}=45 \mathrm{~m})$

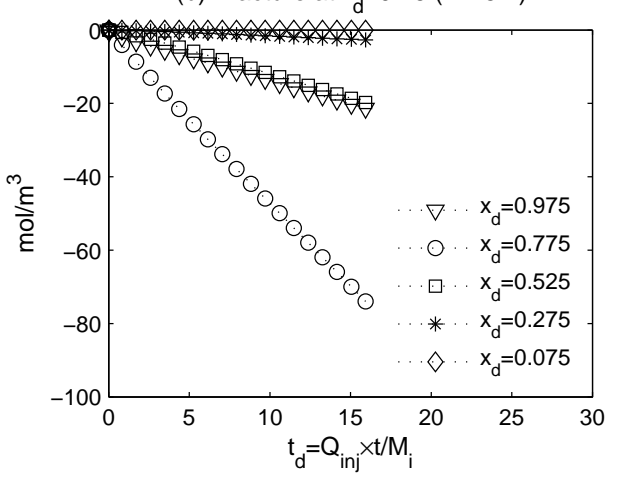

(d) At $x_{d}=0.975, z_{d}=0.45(z=45 m)$

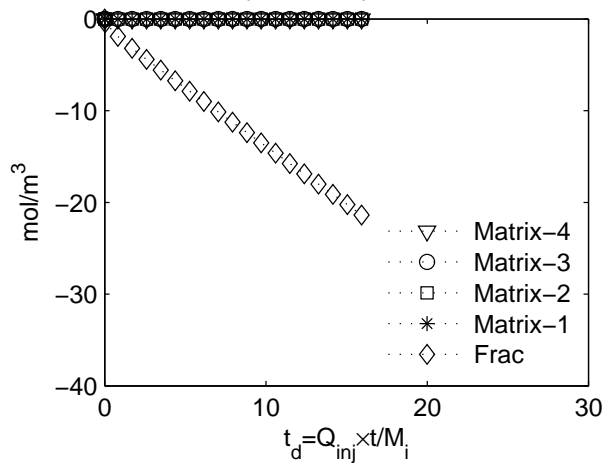

Figure 11: Concentration of aqueous $\mathrm{SiO}_{2}, C_{S i}$ and dissolution of quartz in a chemo-thermo-poro-mechanics simulation. (a) and (c): $C_{S i}^{d}$ at different spatial locations along the fracture medium and $C_{S i}^{d}$ within the fracture medium and rock matrix continua of the injection gridblock. (b) and (d): dissolution of quartz at the locations corresponding to (a) and (c), respectively. $C_{S i, q z, 0}$ is $2.078 \times 10^{-3} \mathbf{~ m o l} / \mathbf{k g}$. 'Frac' implies the fracture medium.
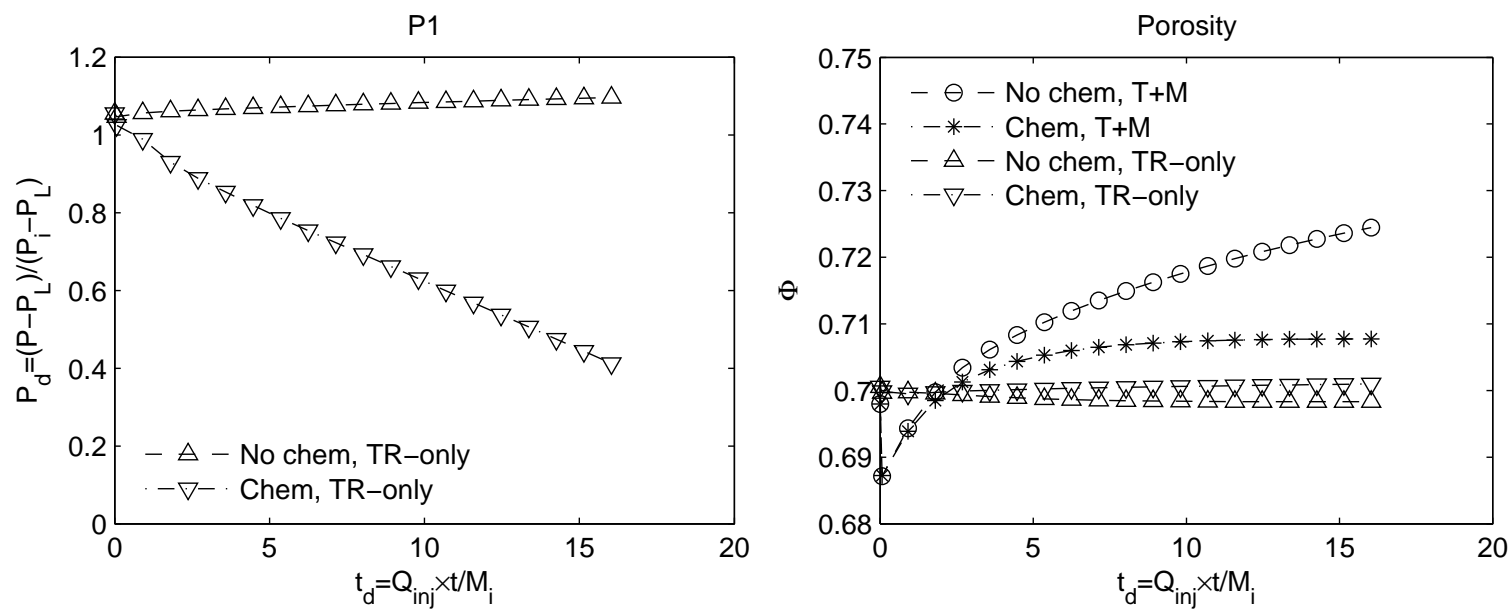

Figure 12: Left: comparison of the pressure results at P1 between with ('Chem') and without ('No chem') reactive transport in TR-only. Right: comparison of porosities among chemo-thermo-poro-mechanics, thermo-poro-mechanics only, and TR only with and without reactive transport. 


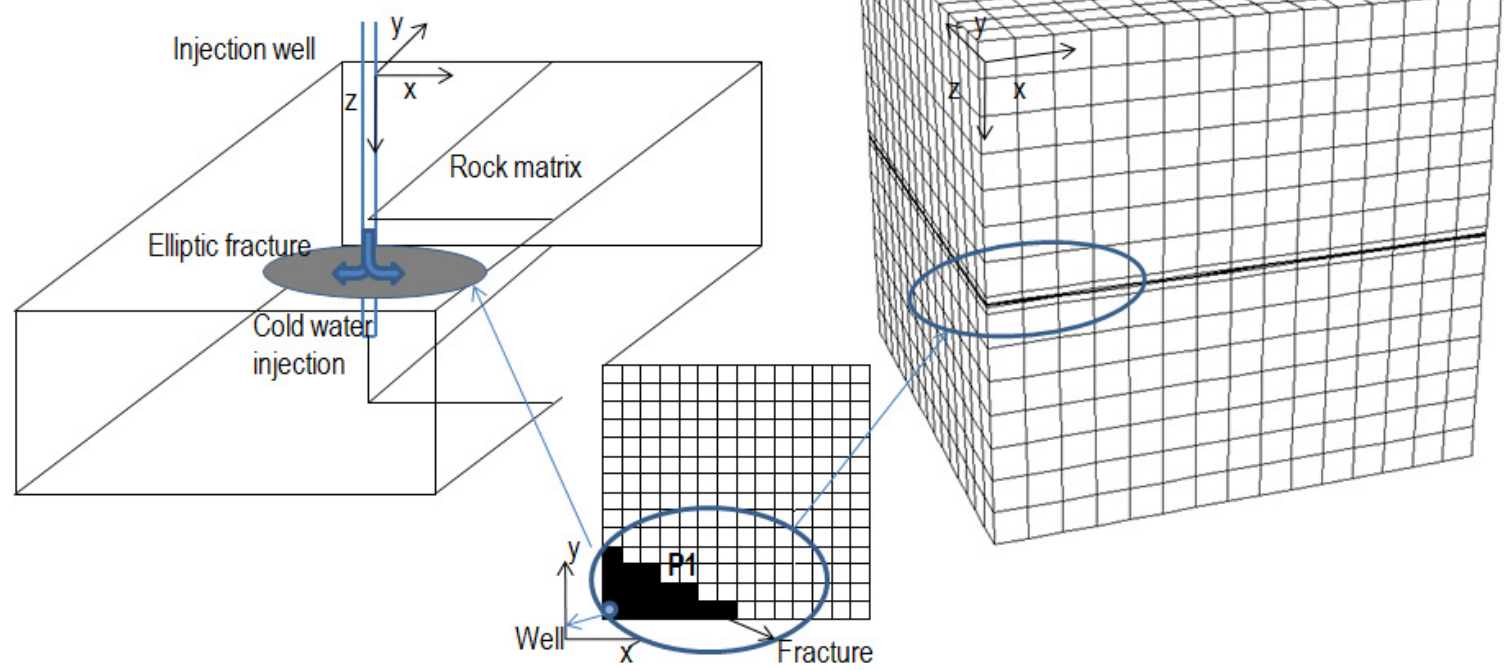

Figure 13: Left: a schematics of a 3D geothermal reservoir with an elliptic fracture. Right: the discretized domain for simulation. The upper and right quadrant is used for the simulation domain.

(a) Chem

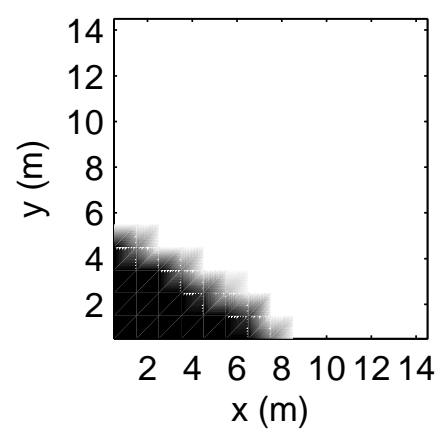

(c) No Chem

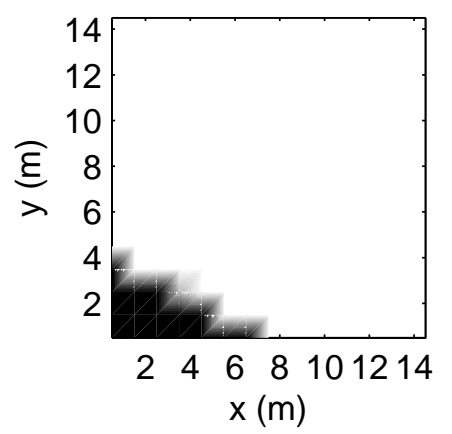

(b) Chem
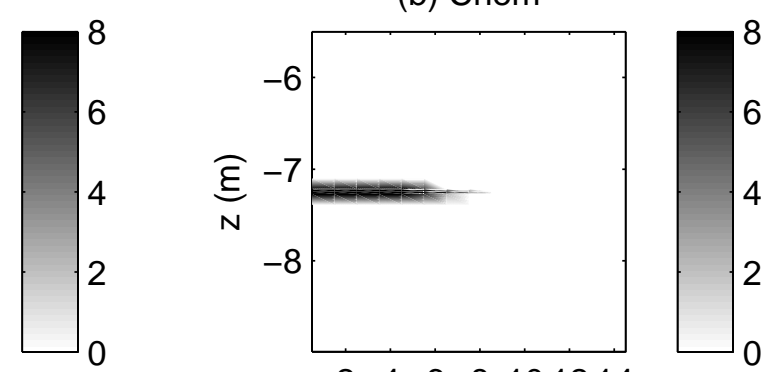

$\begin{array}{lllll}2 & 4 & 6 & 8 & 101214\end{array}$ $x(m)$

(d) No Chem

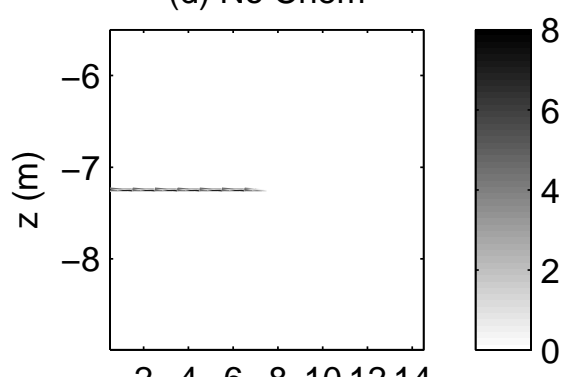

$246 \quad 6 \quad 8 \quad 101214$ $x(m)$

Figure 14: Failure zones at $t_{d}=9.76(\mathbf{t}=\mathbf{1 2 . 1} \mathbf{~ m i n})$ with and without reactive transport at the $10^{\text {th }}$ layer in the $\mathbf{z}$ direction $((\mathrm{a})$ and (c), respectively) and failure zones at the first layer in the y direction ((b) and (d), respectively). Here, $M_{i}=74.24 \mathrm{~kg}$, the initial fluid mass in place at the fracture medium. Values 1-8 indicate the numbers of Gauss points that experience failure, and the initial fractured zone takes 8 , while Values 0 indicates no failed zone. Failed areas with reactive transport are larger than those without reactive transport. 

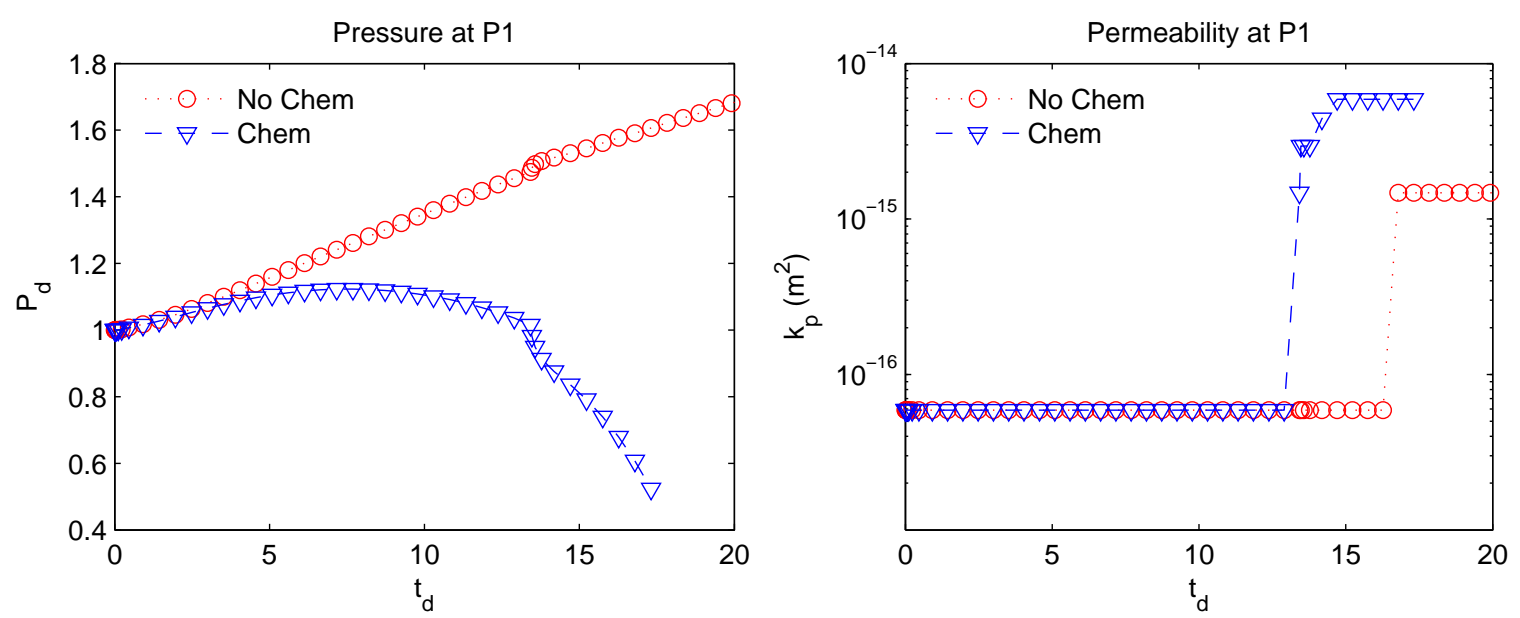

Figure 15: Left: Evolution of pressure (left figure) and permeability (right figure) at P1. Once failure occurs, permeability increases considerably, which affects pressure.
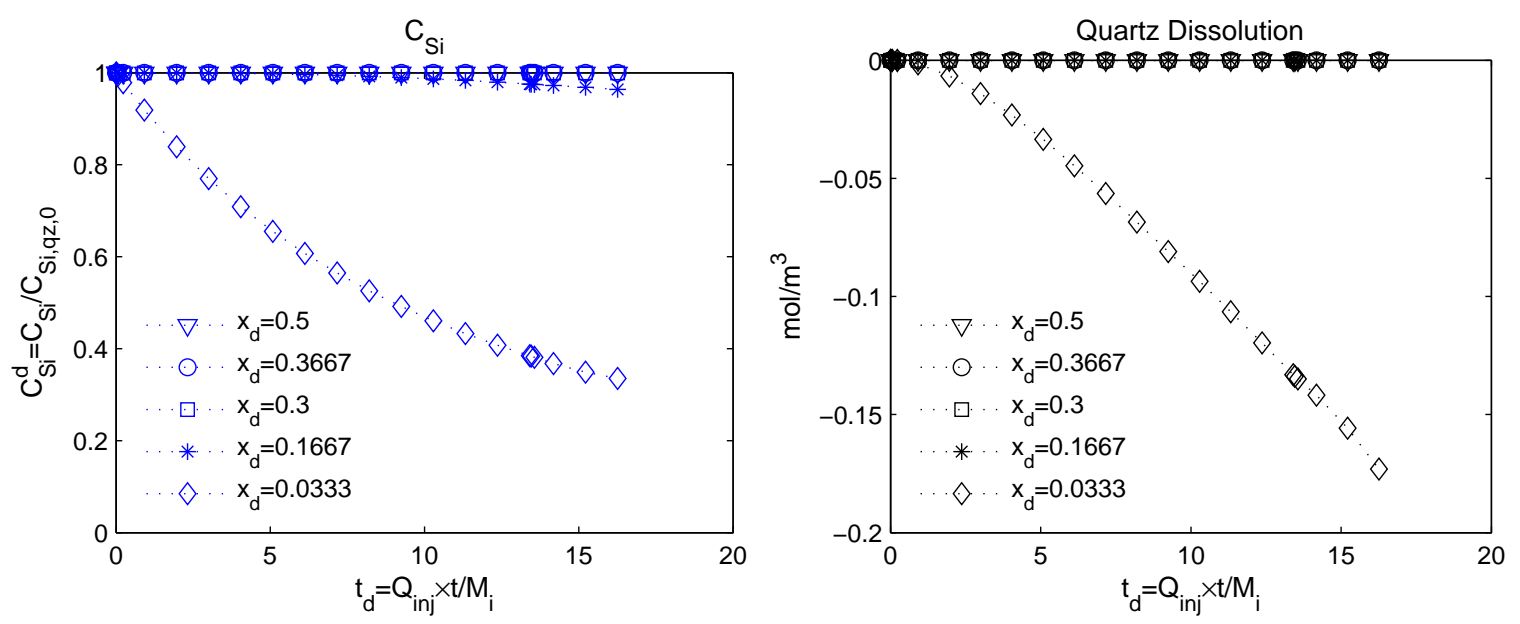

Figure 16: Left: Evolution of $C_{S i}^{d}$ at different locations. Right: Quartz dissolution at different locations. Quartz dissolution occurs as zero $C_{S i}$ of water is injected, and $C_{S i}^{d}$ decreases accordingly. 